\title{
Three-dimensional flow and load characteristics of flexible revolving wings
}

\author{
R. van de Meerendonk ${ }^{1} \cdot$ M. Percin ${ }^{2}$ (D) B. W. van Oudheusden ${ }^{1}$ (D)
}

Received: 19 March 2018 / Revised: 31 August 2018 / Accepted: 5 September 2018 / Published online: 1 October 2018

(c) The Author(s) 2018

\begin{abstract}
The flow field and fluid-dynamic loads of revolving low-aspect-ratio chordwise-flexible wings are studied experimentally at a Reynolds number of 10,000. The investigation involves phase-locked tomographic particle image velocimetry (PIV) complemented with force measurements. The pressure fields are reconstructed from the three-dimensional velocity fields in a complete volume around the wing. For decreasing flexural stiffness, the coherence of this vortex system and spanwise transport of vorticity along the axis of the leading edge vortex (LEV) increase, which contribute to the stability and retention of the LEV. As the LEV low-pressure region becomes smaller with increasing flexibility, the total force on the wing is reduced, while it is tilted towards the lift direction due to the wing deformation. As a result, the drag is significantly suppressed, while the lift remains relatively high. Consequently, the lift-to-drag ratio increases with increasing flexibility and correlates well with the geometric angle of attack. While the sectional lift along the full span is comparable for the different wings, the sectional drag is significantly reduced at the outboard wing for increasing flexibility. The centroids of lift and drag are located at approximately $70 \%$ of the span for all wings throughout the complete revolving motion. Finally, the process of vortex breakdown is found to be related to the formation of a positive spanwise pressure gradient.
\end{abstract}

\section{Introduction}

Bioinspired flapping-wing flight has been of interest particularly in the field of micro-air vehicles (MAVs), since other means of force production (i.e., fixed-wing and rotarywing mechanisms) suffer from the reduced aerodynamic performance at the typical flight regime of MAVs, which is characterized by low Reynolds numbers (on the order of $<10^{4}-10^{5}$ ) (Pines and Bohorquez 2006). Flappingwing flight is a three-dimensional unsteady phenomenon, where the formation of a stable leading edge vortex (LEV) is one of the most prominent mechanisms responsible for the enhanced aerodynamic forces sustaining flapping flight

Electronic supplementary material The online version of this article (https://doi.org/10.1007/s00348-018-2613-1) contains supplementary material, which is available to authorized users.

B. W. van Oudheusden

b.w.vanoudheusden@tudelft.nl

1 Department of Aerospace Engineering, Delft University of Technology, Kluyverweg 1, 2629 HS Delft, The Netherlands

2 Department of Aerospace Engineering, Middle East Technical University, Ankara, Turkey
(Sane 2003). The LEV phenomenon in the context of flapping-wing aerodynamics has been subject to a number of studies in the last 3 decades, particularly to achieve a better understanding of its formation and the mechanisms responsible for its stability. It has been hypothesized that spanwise pressure gradients, the associated vorticity transport (Ellington et al. 1996), and the apparent rotational accelerations which are characterized by the Rossby number (Lentink and Dickinson 2009; Jardin and David 2014, 2015) play an important role in this respect.

In flapping-wing flight, to stay aloft or maneuver, biological flyers drive their wings in three main motions, which are sweeping, pitching, and plunging (heaving). The sweeping (translational) aspect of the full flapping-wing motion can be represented by a revolving wing model, in which the aforementioned rotational inertial mechanisms and spanwise gradients are also present. Accordingly, the flow fields and force generation aspects of revolving low-aspect-ratio wings have been studied for a wide range of parameters as reported in the literature (Jones and Babinsky 2011; Ozen and Rockwell 2012; Venkata and Jones 2013; Garmann et al. 2013; Garmann and Visbal 2014; Percin and van Oudheusden 2015a, b). It was hypothesized that rotational inertial mechanism (Lentink and Dickinson 2009) in combination 
with the spanwise flow (Ellington et al. 1996) is responsible for the prolonged attachment of the LEV and thus augmenting force generation in the case of revolving wings. In this respect, Jardin and David (2014) numerically simulated the flow past a finite-span wing in three configurations: (i) in a uniform free-stream flow, (ii) in a spanwise varying flow, and (iii) in a revolving motion about its root. They showed that a spanwise gradient of the local wing speed leads to stabilization of the LEV but not to enhanced lift, which is observed when including rotational-inertial effects.

The sustained LEV present in a revolving motion is accompanied by a low-pressure region at the suction side of the wing. The associated suction forces acting perpendicular to the wing surface are dominant, such that for a rigid wing the resultant force vector is oriented normal to the wing chord for angles of attack higher than $10^{\circ}$ (Usherwood and Ellington 2002; Birch et al. 2004). Therefore, a direct link between the temporal evolution of the vortical structures and the associated pressure forces can be established when the pressure fields are incorporated in the analysis. In this respect, Tronchin et al. (2015) experimentally studied the loads and pressure field for a flapping wing at a Reynolds number of 1000 using three-dimensional three-component (3D3C) velocity data obtained from phase-averaged crosscorrelated stereo-PIV planes. The acceleration information was determined in an inertial frame by acquiring, per phase angle, 5 volumetric images with small time shifts and subsequently calculating the acceleration from the velocity fields furthest separated in time, with a corresponding temporal resolution of $150 \mathrm{~ms}$. This study suggests that the calculated pressure field is obtained with a sufficient accuracy for a global analysis of the topology of the flow field and the evaluation of loads acting on an immersed object.

An important characteristic of biological flapping-wing flight that is not commonly taken into account in mechanical model simulations like those referred to above is the influence of wing flexibility. Different studies have indicated the possible benefits of flexibility on the aerodynamic performance (Shyy et al. 2010). Combes and Daniel (2003) found that, for biological flyers, the wing size is the dominant factor in the scaling of flexural stiffness and that the spanwise flexural stiffness is 1-2 orders of magnitude higher than the chordwise flexural stiffness. Zhao et al. (2009) studied wings with different chordwise stiffness for different angles of attack at a Reynolds number of 2000 for a constant revolving motion. It was observed that the lift-to-drag ratio is relatively insensitive to wing flexibility for angles of attack from $20^{\circ}$ to $60^{\circ}$, while the ability to generate both lift and drag decreased. Zhao et al. (2011) observed an attached LEV as the dominant flow feature for each wing flexibility; however, the LEV was smaller for lower flexural stiffness (higher flexibility). In addition, the size of the LEV was found to correlate with the aerodynamic forces. Beals and
Jones (2015) studied a two-element revolving wing with passive chordwise flexibility for Reynolds numbers from 10,000 to 25,000 . For the flexible wing, the lift was measured to be consistently lower than that of the rigid wing throughout the revolving motion; however, passive wing deformation mitigated the lift losses when a wake was encountered, i.e., for rotation angles larger than $360^{\circ}$.

The aim of the current study is to investigate the spatial-temporal evolution of the flow field of revolving lowaspect-ratio wings, and to connect the associated vortical structures and pressure fields to the temporal evolution of the fluid-dynamic forces acting on the wing. Wings with different degree of chordwise flexibility are considered, to study the influence of wing deformation on the flow characteristics and force generation performance of the wings. For this purpose, tomographic-PIV measurements were performed to obtain 3D3C flow field data around the complete wing for different phases in the revolving motion. These measurements were acquired with a phase-locked approach, which is explained in more detail in Sect. 2.3. Simultaneous force measurements were carried out by means of a six-component water-submergible force sensor to obtain the temporal variation of the forces during the revolving motion. Pressure fields are reconstructed from the PIV velocity data, which also enables the calculation of forces (both integral and sectional) acting on the wing from the flow fields by use of a control-volume approach.

\section{Experimental methods}

\subsection{Experimental setup and model description}

The experiments were performed in a water tank facility at the Aerodynamics Laboratory of Delft University of Technology (TU Delft), where the revolving motion of the model is controlled by a driving system comprised of a brushed DC motor, a gearbox, a main axis that is mounted vertically in the water tank, and a servo box. The reader is referred to the references (Percin 2015; Percin and van Oudheusden 2015b) for a detailed description of the setup.

The wing model consists of a flat plate with a chord length $(c)$ of $50 \mathrm{~mm}$ and a wing span length $(R)$ of $100 \mathrm{~mm}$, resulting in a wing aspect ratio of 2 . At the leading edge a $1.5 \mathrm{~mm}$ radius, half-round carbon fiber rod is glued on the upper and lower sides of the wing to provide spanwise rigidity. This leading edge structure is clamped in the mount with a $1 \mathrm{~mm}$ offset from the wing root (see Fig. 1). The flexible wing surface is only connected to the rigid leading edge, while the wing root is not connected and, therefore, does not impose a restriction on the wing deformation. The distance between the root and the rotation axis is $42 \mathrm{~mm}$ giving a 


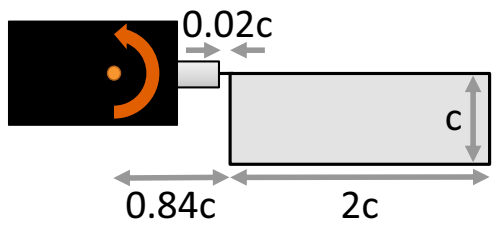

Fig. 1 Schematic view of wing model including the dimensions in terms of the wing chord $(c=50 \mathrm{~mm})$. The wing span length $(R)$ is twice the chord length $(R=2 c)$ and $(r)$ is the position in spanwise direction from the wing root (e.g., $r=0$ at the wing root and $r=R$ at the wing tip)

radius of gyration of $R_{g}=96.4 \mathrm{~mm}$ and a corresponding Rossby number $(R o)$ of approximately $R_{g} / c=1.93$.

The chordwise flexural stiffness is represented by $E I$, where $E$ is the Young's modulus and $I=R h^{3} / 12$ is the area moment of inertia of the wing's cross section along the chord with $h$ being the thickness. The corresponding effective stiffness $\left(\Pi_{1}\right)$ describes the ratio between the elastic bending forces and the fluid-dynamic forces which is evaluated as follows (Shyy et al. 2010):

$\Pi_{1}=\frac{E h^{3}}{12\left(1-v^{2}\right) \rho V_{t}^{2} c^{3}}$,

where $v$ is the Poisson ratio, $\rho$ the density of the fluid, and $V_{t}$ the terminal velocity of the wing. Three wing models with different flexural stiffness have been studied by changing the material and plate thickness as reported in Table 1. In the current study, it is not expected that the difference in wing thickness significantly influences the flow separation and associated LEV formation, because the LE is effectively formed by the mounting structure that is comparable for all three wings (see above).

Although changing the working medium from air to water does not allow a full aeroelastic similarity including inertial effects to be achieved (Percin et al. 2011), the latter is not part of the scope of the present study. This incomplete aeroelastic scaling may be further illustrated as follows. In addition to the Reynolds number $(\mathrm{Re})$ and effective stiffness $\Pi_{1}$, a full aeroelastic scaling involves also the inertia parameter $\Pi_{2}=\frac{\rho_{s}}{\rho_{f}} \frac{h}{c}$ that represents the ratio of inertial to fluid-dynamic forces, where subscripts $f$ and $s$ refer to the fluid and the structure, respectively (Shyy et al. 2010). When changing the medium from air to water while keeping wing dimensions and material properties the same, similarity of Re and stiffness $\left(\Pi_{1}\right)$ can be achieved by appropriately reducing the velocity and simultaneously increasing the wing thickness. Yet, the inertia parameter will be decreased by a factor of about 500 , i.e., the wing behaves as much lighter than in air. Quantifying this effect by taking the dimensions and material properties of the most flexible wing considered in this investigation, we obtain $\Pi_{2} \approx 0.004$ for the current experiment in water, and $\Pi_{2} \approx 2$ for the equivalently scaled wing in air. Thus, inertia effects are relevant in air, but negligible in water experiments. This must be accepted as an unavoidable limitation of air-water flexibility scaling.

\subsection{Motion kinematics}

The $75 \%$ span position of the wing model is taken as the reference position to characterize the motion kinematics. The non-dimensional parameters describing the motion kinematics are: convective time $\left(t^{*}=t \times V_{t} / c\right.$, where $t$ is time in seconds and $V_{t}$ is the constant terminal velocity established after the acceleration phase) and chords traveled $\left(\delta^{*}=\delta / c\right.$, where $\delta$ is the distance traveled by the wing as measured at the reference spanwise position). The revolving wing motion starts from rest (see Fig. 2) and is subjected to a constant acceleration to reach a prescribed $V_{t}$ of $0.2 \mathrm{~m} / \mathrm{s}$ over a time

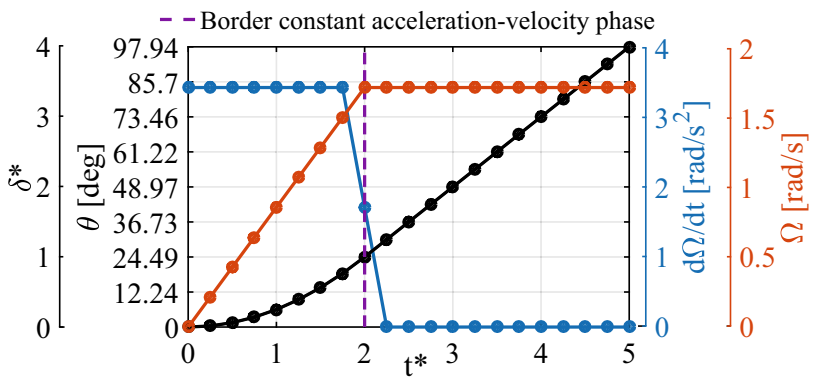

Fig. 2 Wing kinematics: chord distance traveled $\left(\delta^{*}\right)$, rotation angle $(\theta)$, angular velocity $(\Omega=d \theta / \mathrm{d} t)$, and angular acceleration $(d \Omega / \mathrm{d} t)$ as a function of convective time $\left(t^{*}\right)$. Lines connect the discrete measurement points (indicated by the bullets) for visualization purposes
Table 1 Model parameters (PET properties: Hostaphan GN, Mitsubishi polyester film $\mathrm{GmbH})$

\begin{tabular}{llllll}
\hline Description & Material & $\begin{array}{l}\text { Young's modulus } \\
E\left(\mathrm{Nm}^{-2}\right)\end{array}$ & $\begin{array}{l}\text { Thickness } h \\
(\mathrm{~mm})\end{array}$ & $\begin{array}{l}\text { Flexural stiffness } \\
E I\left(\mathrm{Nm}^{2}\right)\end{array}$ & $\Pi_{1}$ \\
\hline Rigid & Plexiglas & $\approx 3300 \cdot 10^{6}$ & 1 & $2.75 \cdot 10^{-2}$ & 65.5 \\
Moderate flexibility & PET & $\approx 4350 \cdot 10^{6}$ & 0.175 & $1.94 \cdot 10^{-4}$ & 0.46 \\
High flexibility & PET & $\approx 4500 \cdot 10^{6}$ & 0.125 & $7.32 \cdot 10^{-5}$ & 0.17 \\
\hline
\end{tabular}

In the definition of $\Pi_{1}$, a Poisson ratio ( $v$ ) of 0.4 for Plexiglas and PET is used. The density of water $(\rho)$ is taken as $1000\left(\mathrm{~kg} / \mathrm{m}^{3}\right)$ 
interval corresponding to a displacement of one chord length (i.e., $0<\delta^{*}<1$ ), after which the wing continues to revolve at a constant velocity (i.e., for $\delta^{*}>1$ ). The accuracy of the brushed DC motor is $0.018 c$ (corresponding to $0.46^{\circ}$ ) in position and $0.0125 V_{t}$ in velocity.

Based on the chord length and the terminal velocity, the Reynolds number is 10,000 . The angle of attack $(\alpha)$ of the plate is $45^{\circ}$. In all the experiments, the complete travel distance is $14 c\left(\delta^{*}=14 c\right)$ corresponding to approximately one full revolution. Although the forces were captured for the full motion, the PIV measurements were limited to the interval $0.0625<\delta^{*}<4$, which covers the evolution until an established flow state is reached. For the rigid case, the temporal resolution (TR) is $62.5 \mathrm{~ms}$ for the entire interval, which corresponds to a non-dimensional temporal resolution $\left(\mathrm{TR}^{*}=\mathrm{TR} \times V_{t} / c\right)$ of 0.25 , resulting in 19 revolving phases. For the flexible wings, the $\mathrm{TR}^{*}=0.25$ for $0.0625<\delta^{*}<1.5$ and $\mathrm{TR}^{*}=0.5$ for $1.5<\delta^{*}<4$, generating a total of 14 revolving phases. The three-dimensional flow fields were ensemble averaged with a sample size of 5 obtained by repeating the measurements for each phase.

\subsection{Volumetric flow imaging by tomographic PIV}

Figure 3 (left) shows a schematic top view of the tomographic-PIV setup. The measurement volume was illuminated by a $200 \mathrm{~mJ}$ double-pulsed Nd:Yag laser at a wavelength of $532 \mathrm{~nm}$. Polyamide spherical particles of $56 \mu \mathrm{m}$ diameter were used as tracer particles. Four 12-bit PCO Sensicam CCD cameras were used to record the particle images. Cameras 1, 3, and 4 have a resolution of $1376 \times 1040$ pixels and a pixel pitch of $6.45 \mu \mathrm{m}$ and are arranged on the same horizontal $x, z$-plane with an aperture angle of $90^{\circ}$. Camera 2 has a resolution of $1280 \times 1024$ pixels and a pixel pitch of $6.7 \mu \mathrm{m}$ and is located above camera 3 with an aperture angle of $20^{\circ}$ with respect to the horizontal plane. Double-frame images are taken at the moment in the motion when the wing is oriented normal to camera 3 and data for different revolving phases are obtained by appropriately changing the starting position of the wing motion, such that the desired phase is reached at the position when the PIV measurement is performed. Each camera was equipped with a Nikon $60 \mathrm{~mm}$ focal objective at a numerical aperture $\mathrm{f}_{\#}=16$. Scheimpflug adapters were used on the three off-axis cameras to align the mid-plane of the measurement volume with the focal plane.

The flow field around the complete wing is captured by combining three tomographic measurement volumes, each measuring $100 \times 75 \times 45 \mathrm{~mm}$ in the $x, y, z$-directions, respectively, as shown in Fig. 3 (right-top). The corresponding magnification factor is approximately 0.09 . The volumes are positioned symmetrically with respect to the mid-span plane of the wing and have an overlap of $5 \mathrm{~mm}(\approx$ six vectors). The illumination volume was kept at a fixed position in the water tank, which corresponds to the nominal position of measurement volume 2 . To change the measurement volume, the complete driving system including wing was translated
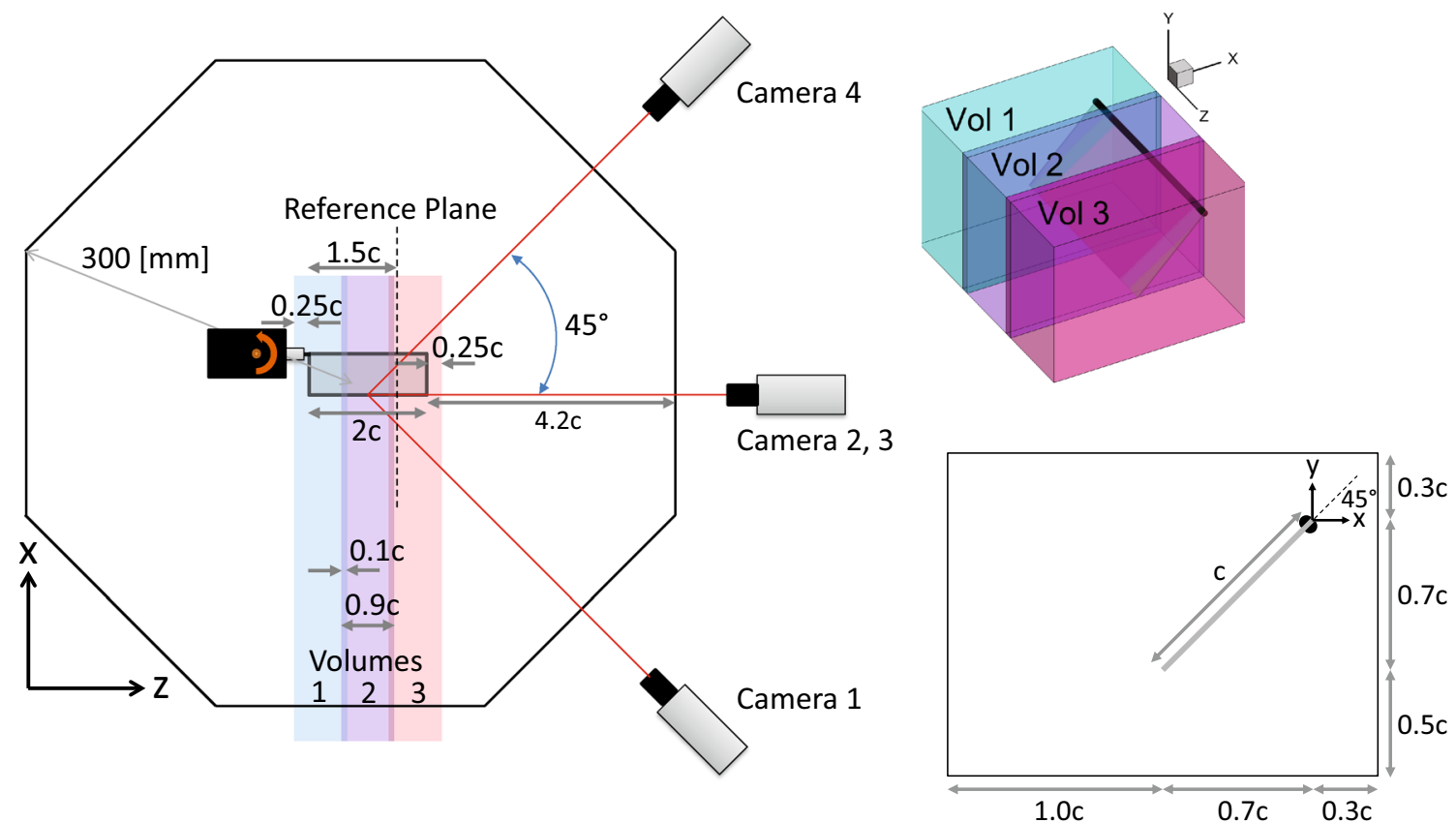

Fig. 3 Left Schematic top view and experimental setup. Right-top Schematic of the measurement volumes. Right-bottom Schematic representation of the chordwise field of view. Schematics illustrated with a rigid wing including its dimensions in terms of its wing chord $(c=50 \mathrm{~mm})$ 
along the direction corresponding to the viewing direction of camera 3 . The image resolution is approximately 13.76 pixels $/ \mathrm{mm}(\approx 688$ pixels $/ c)$. The particle images are interrogated using windows with a final size of $48 \times 48 \times 48$ voxels with an overlap factor of $75 \%$ giving an approximate spatial resolution of $0.87 \mathrm{~mm} /$ vector $(\approx 57$ vectors $/ c)$.

Finally, Fig. 3 (right-bottom) illustrates the wing position with respect to the boundaries of the measurement volumes. The origin of the $x, y$-plane is located at the leading edge with an upstream (positive $x$-direction) and upward (positive $y$-direction) clearance of $0.3 c$ with respect to the upper-right corner of the field of view. The downstream and downward clearance is, respectively, $1.0 c$ and $0.5 c$ from the trailing edge, for the wings, as measured in the undeformed state.

\subsection{Wing reconstruction}

The wing reconstruction is performed using the image of the wing as captured in the PIV recordings and the mapping information that is also used for the tomographic-PIV reconstruction. The chordwise deflection at the tip is reconstructed by defining equidistant points between the leading and trailing edge points along the chord line of the wing in all the images and further triangulation of these points (note that the leading edge point is defined on the wing surface behind the carbon fiber rod). The average uncertainty in the triangulation of the identified points at the tip is approximately $30 \%$ of the vector spacing, resulting in an uncertainty of $0.6^{\circ}$ in the geometric angle of attack.

The angle of attack at the leading edge is set to $45^{\circ}$ by the servo motor at the beginning of the experiment. As the flexible wings deform during the revolving motion under the effect of hydrodynamic loads, this changes the geometric angle of attack as shown for the moderate and high flexibility wings in Fig. 4. For the deformed flexible wings, the geometric angle of attack at a given spanwise location is defined as the angle between the motion direction and the line connecting the reconstructed leading edge and trailing edge points. Subsequently, the twist angle is obtained by measuring the geometric angle of attack at four different spanwise positions, after which a second degree polynomial is fitted to represent the spanwise variation of the geometric angle of attack. The temporal evolution of the wing deformation is given in Fig. 5. At the start of the revolving motion, the deflection of the flexible wings is close to zero, due to the relatively low fluid forces. As the wing velocity increases, the wings start to deflect rapidly until the end of the acceleration phase $\left(\delta^{*}=1\right)$, after which they start to converge to their final wing shape. During the constant revolving motion, the deflection increases approximately linearly along the span from root to tip indicating torsional deformation of the wing and the formation of wing twist. The formed wing twist is relatively low at approximately $2^{\circ}$ and $3.5^{\circ}$ for the
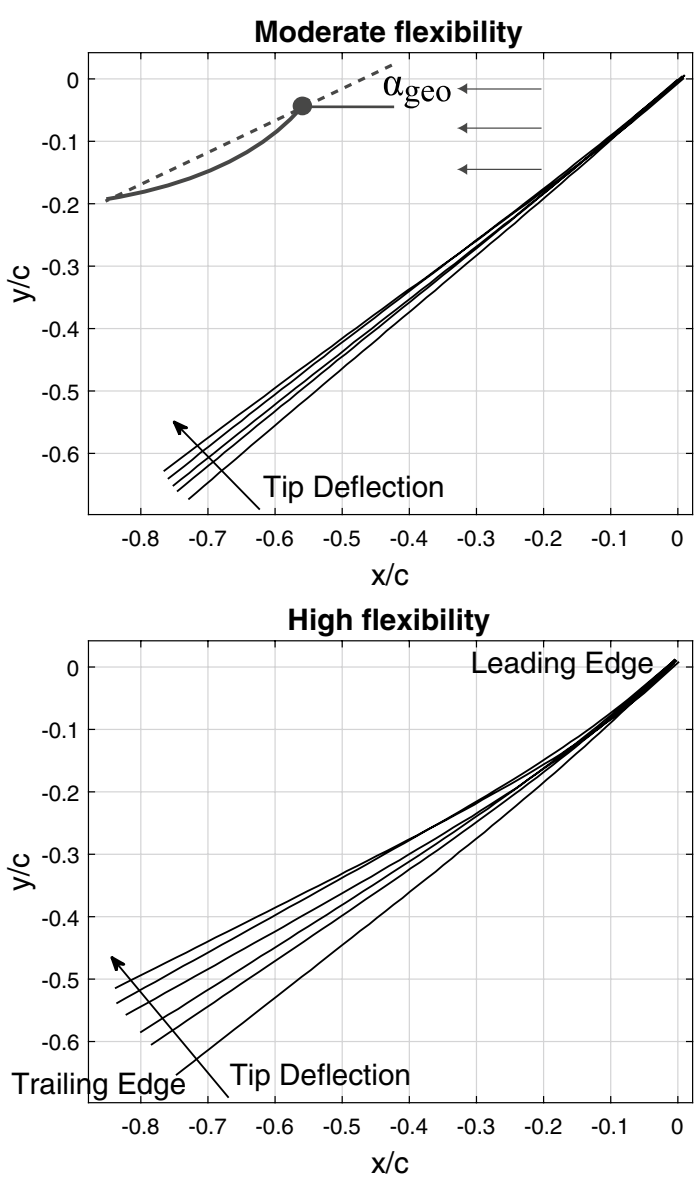

Fig. 4 Tip deformation during revolving motion (the arrows indicate the progression in revolving motion) for moderate flexibility (top) and high flexibility (bottom) wing

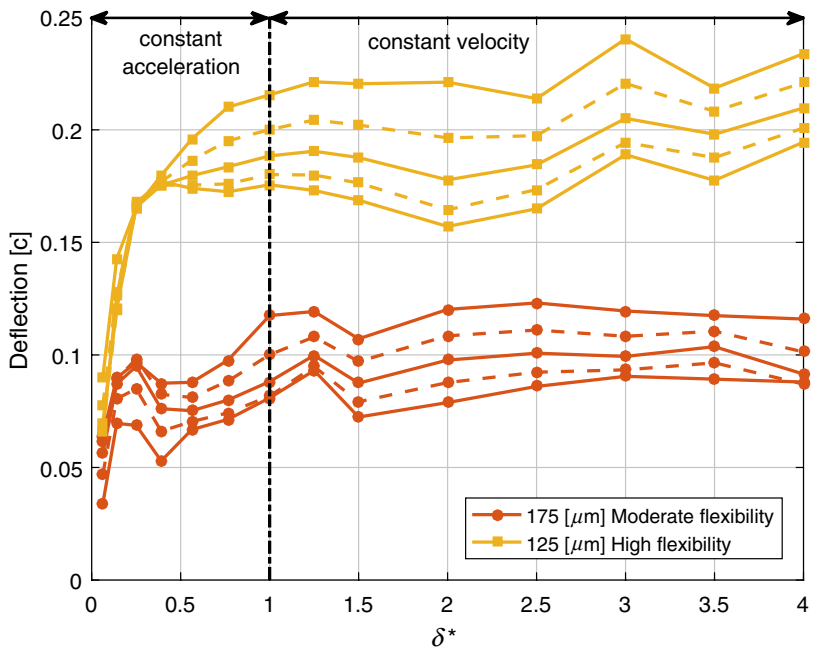

Fig. 5 Temporal evolution of the chordwise deflection in terms of chord length for the moderate and high flexible wing. For increasing deflection, the alternating solid and dashed line style indicate, respectively, $r / R=0$ (root), $r / R=0.25, r / R=0.5, r / R=0.75$ and $r / R=1$ (tip) 
moderate and high flexible wing, respectively. The deflection of the rigid wing is negligible, such that it can be confirmed to behave as indeed fully rigid with a fixed geometric and local angle of attack of $45^{\circ}$ for all the phase angles.

\subsection{Force measurements}

The forces and moments exerted on the wings were measured with a water-submergible ATI Nano17/IP68 force sensor. The sensor is calibrated to have a maximum sensing value of $25 \mathrm{~N}$ in $x$-, $y$-, and $35 \mathrm{~N}$ in $z$-direction with a resolution of $1 / 160 \mathrm{~N}$, and a torque capacity of $250 \mathrm{~N} \cdot \mathrm{mm}$ with a resolution $1 / 32 \mathrm{~N} \cdot \mathrm{mm}$. The force and moment data were acquired at $2 \mathrm{kHz}$ acquisition frequency via a LabVIEW code, which also controls the motors and synchronizes the wing motion with the force data acquisition and the PIV measurements. For ensemble averaging of the force signals, about 260 data records were available for the rigid wing and 200 for each of the flexible wings. From the three different wing models, the test rig for the rigid wing was observed to have the lowest natural frequency, which is $10 \mathrm{~Hz}$. To eliminate electronic noise, the effects of mechanical vibrations from the driving system and resonance of the test rig, the ensemble-averaged force data is additionally filtered with a Chebyshev II low-pass filter that has a cut-off frequency of $8 \mathrm{~Hz}$ with a stopband attenuation of $80 \mathrm{~dB}$. A forward-backward filtering technique is applied to prevent a time-shift of the data. Lift and drag coefficients are defined with respect to the reference velocity, which is the terminal wing velocity at the $75 \%$ span reference plane.

Steady-state conditions are expected to be reached after a sufficiently large rotation angle (Percin and van Oudheusden 2015b; Harbig et al. 2013; Jardin and David 2014, 2015; Beals and Jones 2015; Jones and Babinsky 2011). The measurement uncertainty of the reported lift and drag steady-state value is calculated based on the low-pass-filtered data in the steady-state phase $\left(5<\delta^{*}<10\right)$, providing an approximate relative lift and drag measurement uncertainty of $1.5 \%$ and $1.0 \%$ in terms of the steady-state lift and drag, respectively.

\section{Data processing}

The phase-resolved 3D3C velocity data are used for the analysis of three-dimensional coherent flow structures. In this respect, isosurfaces of $Q$-criterion are used to visualize the vortical structures (Hunt et al. 1988). Although this property is not rotation invariant, i.e,. the components $\mathrm{d} u_{x} / \mathrm{d} z$ (variation of $x$-oriented velocity component in spanwise direction) and $\mathrm{d} u_{z} / \mathrm{d} x$ (variation of spanwise velocity component in $x$-direction) are different in the rotating reference frame compared to the inertial reference frame when revolving around the $y$-axis, the identified $Q$-criterion isosurfaces are very similar and the differences are negligible. In some of the figures, the vortical structures are colored by helical density, $h=\boldsymbol{u} \cdot \boldsymbol{\omega}$ with $\boldsymbol{u}$ being the velocity vector and $\boldsymbol{\omega}$ the vorticity vector (Moffatt 1969). Helicity is indicative of the vorticity flux along the primary axis of the vortex and is calculated in the inertial reference frame, such that the vorticity flux along the primary axis of a vortex is indicative of the spanwise and chordwise advection of vorticity.

The instantaneous pressure field reconstruction is computed from the phase-resolved 3D3C velocity data under the assumption of incompressible flow, employing the spatial integration of the Navier-Stokes equations (van Oudheusden 2013), while the sectional loads are reconstructed using a control-volume approach (Anderson 2011). The experiments were performed in water with a constant density $(\rho)$ of approximately $1000 \mathrm{~kg} / \mathrm{m}^{3}$ and dynamic viscosity $(\mu)$ of approximately $1 \times 10^{-3} \mathrm{~N} \cdot \mathrm{s} / \mathrm{m}^{2}$. At low Reynolds numbers, the flow is very repeatable for the same kinematic motion, which justifies the use of phase-locked measurements (Poelma et al. 2006; Percin and van Oudheusden 2015a; Percin 2015). In view of this phase-locked measurement procedure, the pressure reconstruction is most conveniently setup in the rotating reference frame aligned with the revolving wing, regarding the evaluation of the temporal derivatives from the phase-locked flow field data sets. Note that, as the pressure is a scalar quantity, the reconstructed pressure is independent of the reference frame (inertial or moving) that is used (Vanyo 1993).

The flow fields acquired in the PIV measurements are obtained in the inertial laboratory reference frame. The velocity field data are subsequently converted to the rotating reference frame. Assuming incompressible flow, a constant viscosity and discarding gravity, the pressure gradient in the rotating reference frame is evaluated as follows:

$$
\begin{aligned}
\frac{1}{\rho} \nabla p= & -\frac{D \boldsymbol{u}}{D t} \\
& -\underbrace{\boldsymbol{\Omega} \times\left(\boldsymbol{\Omega} \times \boldsymbol{r}_{\mathrm{pv}}\right)}_{\text {Centrifugal }}-\underbrace{2 \boldsymbol{\Omega} \times \boldsymbol{u}}_{\text {Coriolis }}-\underbrace{\frac{d \boldsymbol{\Omega}}{d t} \times \boldsymbol{r}_{\mathrm{pv}}}_{\text {Euler }}+\nu \nabla^{2} \boldsymbol{u},
\end{aligned}
$$

where $\boldsymbol{u}$ is the velocity vector in the moving reference frame, $\boldsymbol{\Omega}$ the angular velocity of the wing, $\boldsymbol{r}_{\mathrm{pv}}$ the position vector measured from the rotation axis $(0.84 c$ offset in $z$-direction with respect to the root of the wing, as depicted in Fig. 1), $p$ is the pressure, and $v$ is the kinematic viscosity. The centrifugal, Coriolis, and Euler effects introduce apparent forces due to the non-inertial reference frame. The material derivative $D \boldsymbol{u} / D t$ is calculated according to the Eulerian approach, by separately evaluating the local time-derivative and convective terms. The spatial and temporal derivatives are calculated with a second-order-accurate central-difference scheme, except at the boundaries of the temporal evolution 
where a first-order-accurate difference scheme is employed. Subsequently, the pressure gradient field is integrated by setting up a Poisson problem, which is discretized using a finite second-order-accurate central-difference scheme resulting in a seven-point stencil. At the boundaries of the integration domain, Neumann boundary conditions (spatial gradient of pressure) normal to the boundary are imposed. The Neumann boundary conditions are set up using a second-orderaccurate central-difference scheme by introducing ghost points outside of the calculation domain.

The integrated pressure, obtained by means of the Poisson problem using Neumann boundary conditions on all boundaries, gives the pressure up to an arbitrary constant which is different for the three volumes. To obtain a smooth transition between the volumes, the pressure fields are adjusted with respect to volume 2 at the mid-planes of the overlap regions from volume 1-2 and volume 3-2 (as depicted in Fig. 3). Subsequently, the complete pressure field is expressed with respect to a reference pressure of 0 $\mathrm{Pa}$, which is defined in the lower right corner of the flow field (maximum $x$-location; minimum $y$-location) in which the flow is assumed to be undisturbed.

The LEV circulation is calculated based on the spanwise component of vorticity $\left(\Gamma_{\mathrm{LEV}}=\iint_{S_{x y}} \omega_{z} d x d y\right)$ and it is non-dimensionalized by $V_{t}$ and $c\left(\Gamma_{\mathrm{LEV}}^{*}=\Gamma_{\mathrm{LEV}} /\left(V_{t} c\right)\right)$. Moreover, the vorticity flux is calculated at several chordwise-oriented planes $\left(q_{\mathrm{LEV}}=\iint_{S_{x y}} \omega_{z} U_{z} d x d y\right)$ and normalized by $V_{t}^{2} c$. In these calculations, the integration area $\left(S_{x y}\right)$ is defined as the region for which $\gamma_{2}>2 / \pi$ as described by Graftieaux et al. (2001). The in-plane coordinates of the LEV centroid are acquired by calculating the center of either spanwise vorticity or the pressure distribution in the integration region $S_{x y}$.

The sectional forces are estimated by evaluating the integral form of Eq. 2 in a thin-sectional control volume with dimensions matching the field of view in the $x$ - and $y$-directions and a spanwise thickness of $d z$ in the $z$-direction that is centered at a given spanwise plane. Then, sectional lift and drag coefficients for that spanwise plane are defined as follows:

$C_{l(d)}=\frac{L^{\prime}\left(D^{\prime}\right) / \mathrm{d} z}{\frac{1}{2} \rho V_{t}^{2} c}=\frac{l(d)}{\frac{1}{2} \rho V_{t}^{2} c}$,

where $L^{\prime}$ and $D^{\prime}$ are the lift and drag calculated from the sectional control-volume approach.

All results in this paper concerning the temporal derivative in the evaluation of the pressure fields and fluid-dynamic forces are consistently given for $\mathrm{TR}^{*}=0.5$ and it was verified that using higher temporal data $\left(\mathrm{TR}^{*}=0.25\right)$ when available did not provide significantly different results.

Due to the presence of the wing in the measurement region, the reconstructed velocity vectors at and in the close vicinity of the wing have a relatively high uncertainty and need to be excluded from the pressure reconstruction. Based on the position information obtained from the wing reconstruction, a mask is defined around the wing. By means of a mask convergence study, the optimum mask size, defined as the smallest size for which converged lift and drag values are obtained, was determined to be seven vectors, as illustrated in Fig. 6.

\section{Results}

Within the current investigation of the three-dimensional flow and load characteristics of chordwise-flexible wings, first, the hydrodynamic loads are analyzed and discussed (Sect. 4.1). Next, the comparison of the three-dimensional flow fields is given (Sect. 4.2), followed by an investigation of the force production mechanisms in which the reconstructed threedimensional pressure fields in relation to the coherent vortical structures and the spanwise variation of the forces are discussed (Sect. 4.3). Finally, the relation between the pressure gradient and the LEV behavior is investigated (Sect. 4.4).
Fig. 6 Mask convergence study of the rigid wing for different mask vector offset values $(N)$, calculated with a temporal resolution of $\mathrm{TR}^{*}=0.5$. Left Temporal evolution of lift coefficient. Right Mask implementation
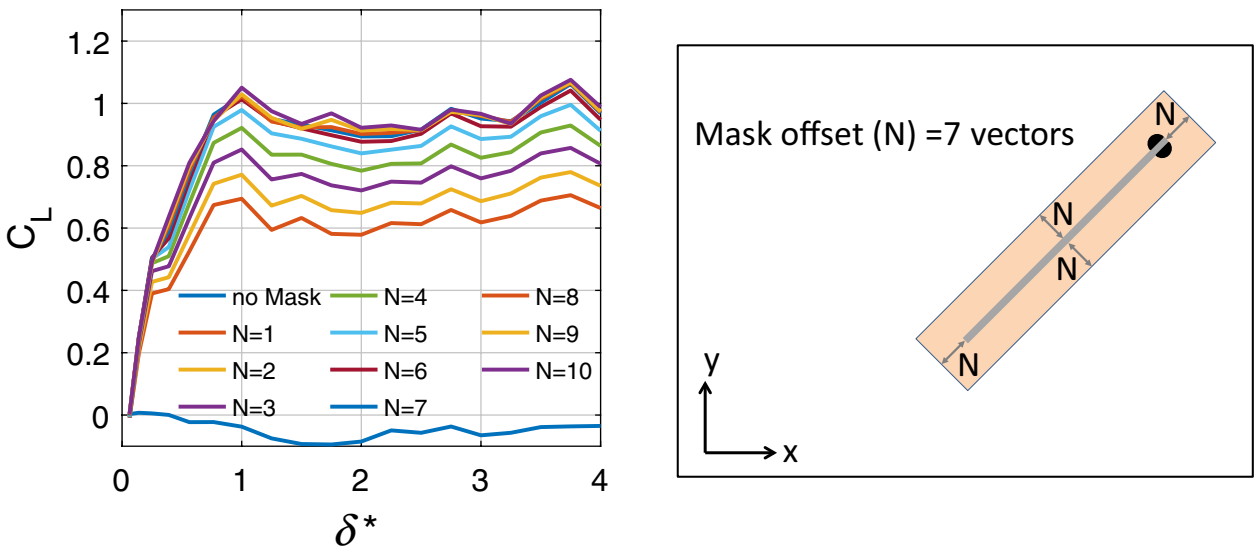


\subsection{Hydrodynamic loads}

In this section, the force generation characteristics of the different flexible wings are investigated in conjunction with their deformed shapes. First, the force measurements are given, followed by the relationship of the force scaling with the geometric angle of attack.

\subsubsection{Force measurements}

The direct force measurements reveal that the drag and lift for the rigid wing are comparable (see Fig. 7). With the angle of attack being $45^{\circ}$, this confirms that the pressure forces are dominant, such that the net force vector is oriented normal to the wing surface. This has also been confirmed in separate force measurements performed in a similar experimental configuration $(\mathrm{Ro}=1.66)$ with revolving-surging flat plates undergoing the same motion kinematics $(R e=10,000)$ at angles of attack in the range of $7^{\circ}$ to $100^{\circ}$, as shown in Fig. 8(a). The results of this study are also compared with those reported by Birch et al. (2004) for a wing model with the planform of a Drosophila wing at two different Reynolds numbers. Although the wing planforms are different, the variation of the force vector angle displays a very similar trend for the cases of $R e=1400$ (Birch et al. 2004) and the present experiments $(R e=10,000)$, such that the force vector is approximately normal to the wing surface for angles of attack greater than $15^{\circ}$. This supports the conclusion that the separated flow at the leading edge, which leads to the formation of an LEV with its associated low-pressure region, becomes the dominant force generation mechanism under these conditions.

For all wing cases, the forces build up rapidly at the start of the motion due to non-circulatory added mass effects. For a thin wing, the added mass reaction force acts normal

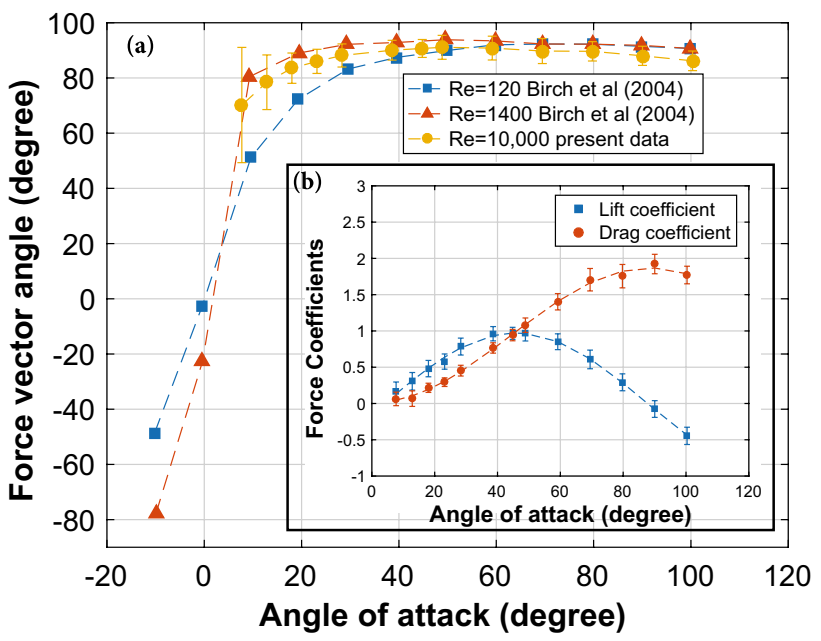

Fig. 8 Rigid revolving wing at steady-state conditions, defined as the average forces in the motion period of $5<\delta^{*}<10$ with $R o=1.66$. a Force vector angle comparison with Birch et al. (2004). b Curve fit of lift and drag coefficients as a function of angle of attack for the case: " $R e=10,000$ present data"

to the local wing surface and is proportional to the acceleration component in this direction (Pitt Ford and Babinsky 2013). Therefore, for the rigid wing, its contribution remains constant throughout the acceleration phase. However, for the flexible wings, the acceleration component in the wing-normal direction changes, since the wings deform, reducing the effective wing angle, and so does the magnitude of the added mass force. In addition to the added mass reaction force, circulatory forces associated with the generation of the LEV build up gradually with increasing velocity. At the end of the acceleration phase at $\delta^{*}=1$, there is a slight decrease in the force components as the added mass effect vanishes. Following the acceleration phase, lift and drag continue to increase until a maximum is reached at approximately $\delta^{*}=4.5$ for constant acceleration ; constant velocity
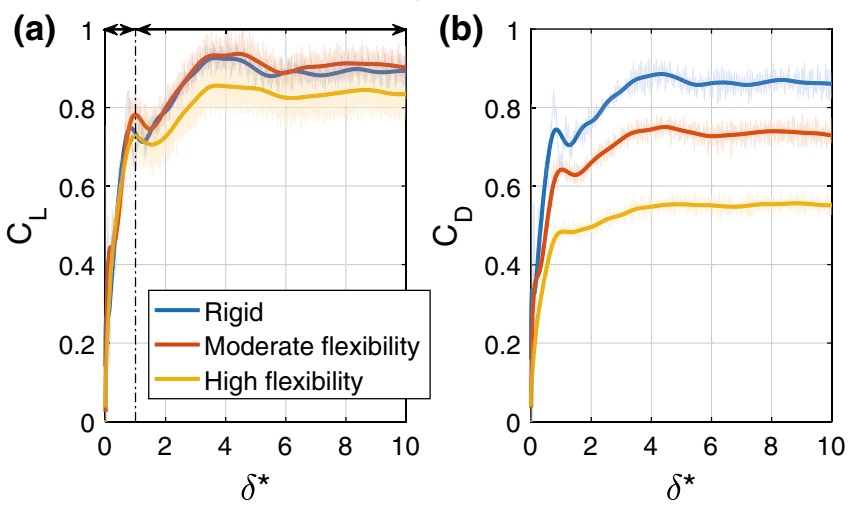

(c)

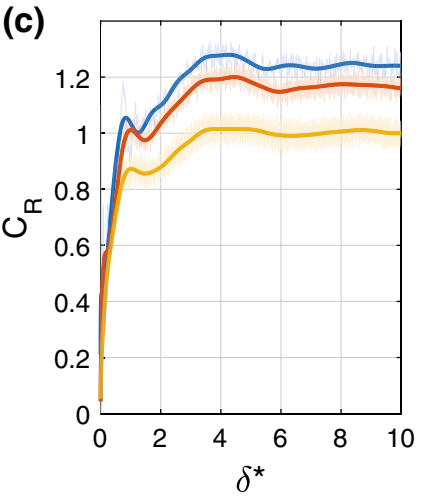

Fig. 7 Temporal evolution of the measured $\mathbf{a}$ lift, $\mathbf{b}$ drag, $\mathbf{c}$ resultant force $\left(C_{R}=\sqrt{C_{L}^{2}+C_{D}^{2}}\right)$, and $\mathbf{d}$ lift-to-drag ratio for different flexibilities. The light background signal is the ensemble average of the raw force data, and the thick solid line is the corresponding low-pass-filtered data 
Table 2 Comparison of the steady-state geometric angle of attack (acquired from the force relation shown in Eq. 4 and optical wing reconstructions), and lift-and-drag coefficients for the rigid and chordwise-flexible wings

\begin{tabular}{|c|c|c|c|c|c|c|c|c|c|}
\hline & \multicolumn{3}{|c|}{ Balance data $\left(\alpha_{\text {geo }}\right.$ via Eq. 4$)$} & \multicolumn{6}{|c|}{ Wing reconstruction data (force predictions via Eqs. 5 and 6) } \\
\hline & \multirow[b]{2}{*}{$\alpha_{g e o}(\mathrm{deg})$} & \multirow[b]{2}{*}{$C_{L}$} & \multirow[b]{2}{*}{$C_{D}$} & \multicolumn{3}{|l|}{ Root } & \multicolumn{3}{|l|}{ Tip } \\
\hline & & & & $\alpha_{g e o}(\mathrm{deg})$ & $C_{L}$ & $C_{D}$ & $\alpha_{\text {geo }}(\mathrm{deg})$ & $C_{L}$ & $C_{D}$ \\
\hline Rigid & 43.7 & 0.89 & 0.86 & 45 & 0.97 & 0.96 & 45 & 0.97 & 0.96 \\
\hline Moderate flexibility & 38.6 & 0.90 & 0.73 & 41 & 0.96 & 0.83 & 39 & 0.94 & 0.76 \\
\hline High flexibility & 32.6 & 0.84 & 0.55 & 34.5 & 0.88 & 0.62 & 31 & 0.82 & 0.52 \\
\hline
\end{tabular}

Steady-state balance measurements averaged over the range $5<\delta^{*}<10$ (Fig. 7) are compared with the reconstructed steady-state force for a rigid wing that revolves at the same geometric angle of attack

all the cases. For the rigid wing case, similar results have been reported by Percin and van Oudheusden (2015a), Jones et al. (2016a). Subsequently, the forces decrease slightly for all wings until nearly steady-state conditions are reached at approximately $\delta^{*}=5$.

The comparison of the lift data shows that the lift generation of the rigid and moderate flexible wings is comparable, while smaller lift levels are achieved in the high flexibility case. The drag shows a monotonic decrease with decreasing flexural stiffness. At steady-state conditions, for $\delta^{*}=10$, the lift coefficient of the high flexible wing is approximately $7 \%$ lower compared to the rigid wing, while the lift of the moderate flexible wing is approximately $1 \%$ higher. The steady-state drag of the moderate and high flexible wings is, respectively, $15 \%$ and $36 \%$ lower compared to the rigid wing, which results in an increased lift-to-drag ratio of approximately $18 \%$ and $45 \%$, respectively (Fig. 7 d). The total resultant force (Fig. 7c) acting on the model is decreased with decreasing flexural stiffness throughout the revolving motion. For steady-state conditions, the resultant force of the moderate and high flexibility is, respectively, $6 \%$ and $19 \%$ lower compared to the rigid wing.

\subsubsection{On the relationship of the force scaling with geometric angle of attack}

As discussed in the previous section, the forces on the wing are dominated by the pressure forces associated with the LEV suction, and as a result the net force is oriented normal to the wing surface. In view of this, the geometric angle of attack of a rigid wing can be related to the drag-to-lift ratio (Usherwood and Ellington 2002) as follows:

$\alpha_{\text {geo }}=\tan ^{-1}(D / L)$.

However, flexible wings deflect under the action of the fluid-dynamic forces, which leads to a deviation of the net force vector orientation with respect to the angle of attack that is initially set (Zhao et al. 2009). Based on the lift-todrag ratio, the effective geometric angles of attack $\alpha_{g e o}$ at approximately steady-state conditions $\left(\delta^{*}=4\right)$ for the rigid, moderate flexible, and high flexible wings are calculated using Eq. 4. These are compared in Table 2 with the actual geometric angle of attack values obtained from the optical wing reconstruction results (see Sect. 2.4). Furthermore, to investigate to what extent the geometric angle of attack of the wing, as acquired from the reconstructed wing shapes, can represent the forces of the deformed wing, a comparison is made with the forces experienced by a rigid wing at the same angle of attack. The empirical relation between the steady-state forces and the (geometrical) angle of attack is obtained from additional force measurement results for a rigid revolving wing (see Sect. 4.1.1). The corresponding relations for the steady-state lift and drag as a function of the angle of attack for the rigid revolving wing, see Fig. 8b, are described by the following curve fit:

$C_{L}=0.016-0.16 \cos (0.038 \alpha)+0.94 \sin (0.038 \alpha)$

$C_{D}=0.941-0.93 \cos (0.036 \alpha)-0.006 \sin (0.036 \alpha)$.

Although the wing reconstruction is an approximation of the true wing shape, it can be observed that the reconstructed geometric angles of attack of the wing agree reasonably well with the direction of the net force vector for the studied wings. Moreover, there is a reasonable agreement between the actual forces and the estimations following from the empirical relation and the reconstructed wing angles. These observations suggest that, for the chordwise-flexible wings considered in this study, the geometric angle of attack is representative for the net force acting on the model at steady-state conditions, as illustrated in Fig. 9.

\subsubsection{Conclusion}

The force measurements show that, during the acceleration phase, the build-up of lift is similar, while the buildup of drag is significantly lower for increasing flexibility. At steady-state conditions, the lift is similar for the rigid and moderate flexible wing, while it is only slightly lower (about 7\%) for the high flexible wing. The drag decreases 


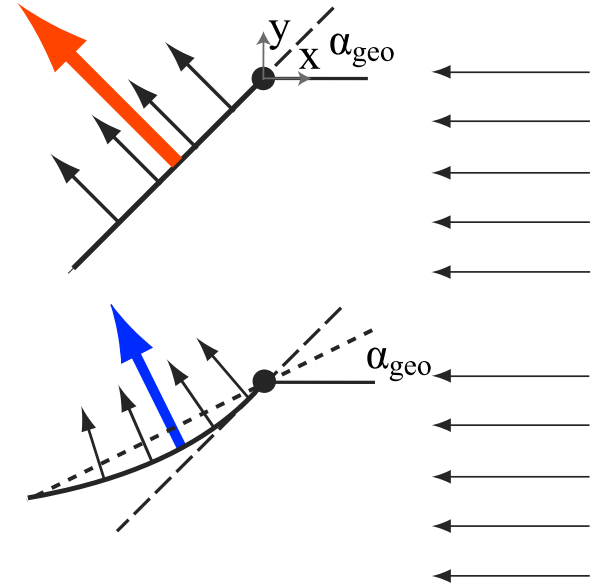

Fig. 9 Net force angle in relation to the geometric angle of attack for the rigid wing (top) and chordwise-flexible wing (bottom). Relative reference flow is from right-to-left, small vectors represent local net forces that act normal to the wing, and the large vector represent the resultant force monotonically with increasing flexibility, such that the liftto-drag is increased significantly (up to $45 \%$ for the high flexible wing). In addition, it is shown that a rigid wing with a geometric angle of attack identical to that of the deformed chordwise-flexible wing generates similar lift and drag, which suggests that, at steady-state conditions, the geometric angle of attack is representative for the resultant force.

\subsection{Three-dimensional flow fields}

In the presentation of the three-dimensional flow fields, first, the temporal development of the coherent structures, the spanwise vorticity, and the spanwise flow are discussed. Next, the spanwise wing characteristics in terms of the LEV properties are discussed.

\subsubsection{Temporal development of coherent structures}

Coherent flow structures for the rigid wing at four phases of the revolving motion are depicted in Fig. 10 by means of isosurfaces of the $Q$-criterion and non-dimensional spanwise vorticity contours $\left(\omega_{z} c / V_{t}\right)$ at several spanwise locations and
Fig. 10 Temporal evolution of vortical structures for the rigid wing. Top two rows Vortical structures for different isosurfaces of Q-criterion, top view, and side view: $Q /(V t / c)^{2}=3$ (White), $Q /(V t / c)^{2}=10$ (Orange), and $Q /(V t / c)^{2}=30$ (Pink). Bottom two rows Spanwise vorticity contours $\left(\omega_{z} c / V_{t}\right)$ along span and at $r / R=0 / 5$

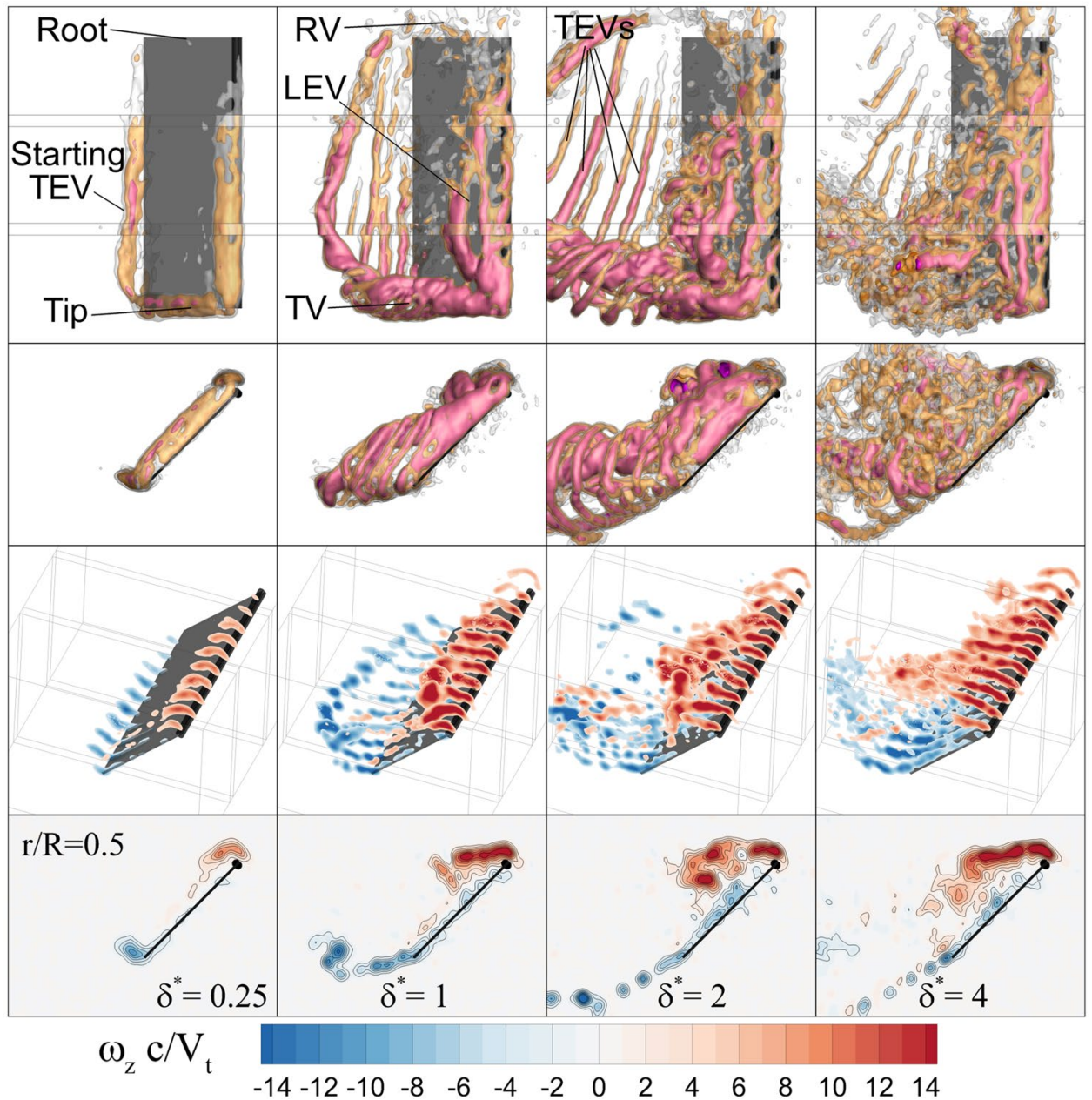


at the mid-plane of the wing, whereas the complete evolution throughout the revolving motion is provided in the figure attached as the Online Resource 1.

The PIV measurements reveal a vortex system consisting of a leading edge vortex (LEV), a trailing edge vortex (TEV), a tip vortex (TV), and a root vortex (RV) that start to develop from the onset of the motion. The LEV forms at the start of the revolving motion (see $\delta^{*}=0.25$ ) and initially displays approximately two-dimensional characteristics, which can be evidenced from the spanwise vorticity contour plots (third row in the figure). At this time step, the flow field also contains a single TEV (i.e., starting vortex) and a coherent TV which does not show any variations along the chord of the wing. The topography of the LEV changes as the motion continues in the acceleration phase, such that it increases in size toward the tip of the wing in accordance with the increasing velocity. It also develops a fragmented form with the initial LEV lifted off and tilted in the downstream direction at the outer half of the span, which is clearly visible at the time step of $\delta^{*}=1.0$. The starting TEV advects downstream connected to the TV which is lifted off from the wing surface. There are secondary smallscale TEVs extending along the complete span of the wing, which are connected to swirling features of the TV, which were also reported by Percin and van Oudheusden (2015a). After the acceleration phase $\left(\delta^{*}>1.0\right)$, the flow structures continue to grow in size with the LEV forming an archshaped bubble-like structure (see $\delta^{*}=2$ ), as also reported by Garmann et al. (2013), Carr et al. (2013), Percin and van Oudheusden (2015a). Near mid-span $(r / R=0.5)$, the core of the LEV is significantly expanded into the bubble-like structure which has two legs pinned to the wing surface at the tip and at about $r / R=0.4$. This observation is in agreement with Fig. 14 (see Sect. 4.2.3) where the minimum distance of the LEV to the wing surface, as expressed by $s / c$, at the tip and at about $r / R=0.4$, confirms the two pinned legs. In the subsequent phases of the motion, this arch-shaped structure extends downstream with the outer leg attachment point moving toward the trailing edge progressively, indicating the onset of vortex breakdown, giving way to the formation of small-scale vortical structures and a chaotic less-coherent flow field particularly at the outboard locations of the revolving wing. Eventually, at $\delta^{*}=3.5-4.0$, the process of vortex bursting is completed and approximate steady-state conditions are reached, which is in accordance with the findings of Jones et al. (2016b). As evident from the spanwise vorticity contour plots depicted at the mid-span of the wing, the LEV gradually moves towards the trailing edge and it starts to interact with the TEV. As a result, smallscale positive vorticity pockets are shed from the trailing edge together with the small-scale train of TEVs of negative vorticity. As hypothesized by Garmann and Visbal (2014), this phenomenon can be put in relation to the evolution of the unsteady forces.

In Fig. 11, three-dimensional vortical structures are compared for the three wings of different flexibility at $\delta^{*}=1.0$ and $\delta^{*}=1.5$. The global topology of the vortex system for the three wing models is similar, although the orientation of the structures is clearly affected by the deformation of the wing structure, which can be evidenced from the side

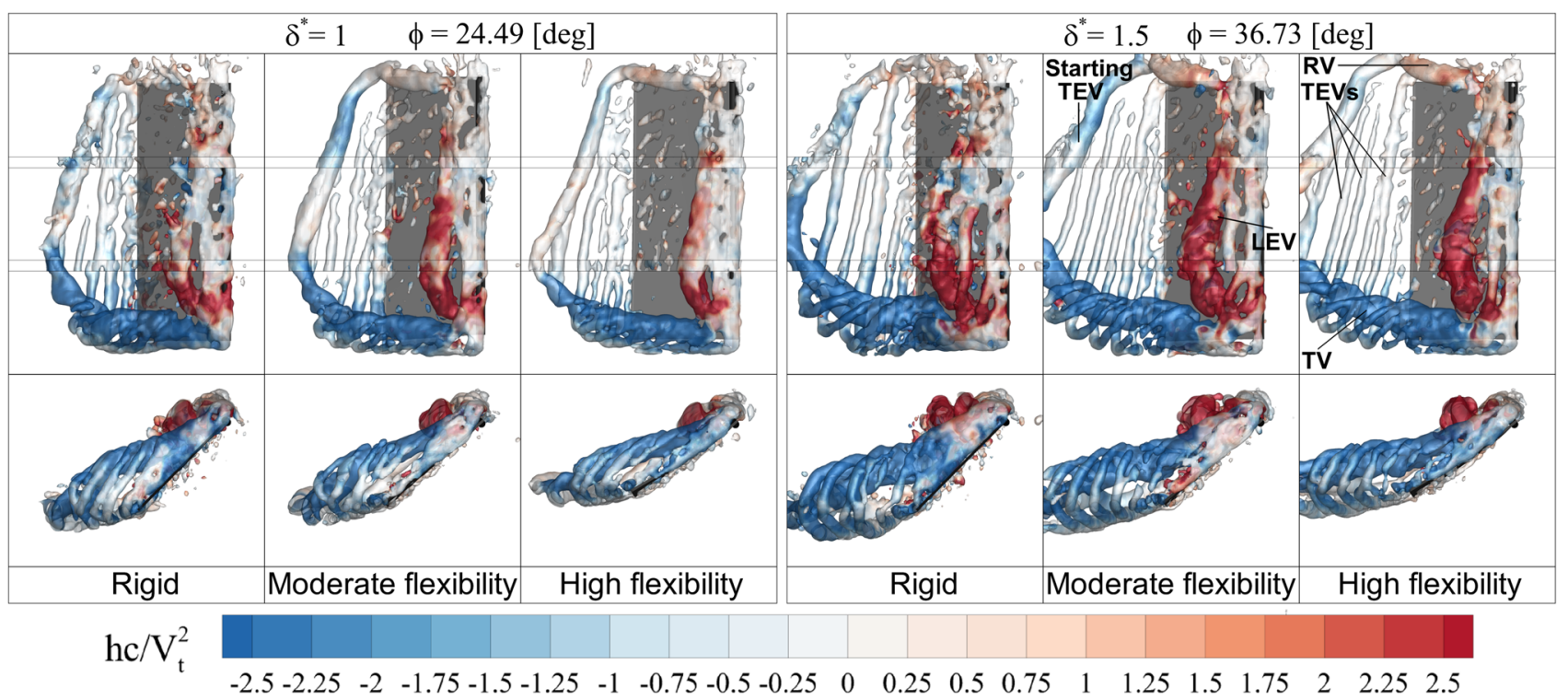

Fig. 11 Vortical structures colored by helical density. Isosurfaces of $Q$-criterion: $Q /\left(V_{t} / c\right)^{2}=3$ (White) colored by helical density $\left(h c / V_{t}^{2}\right)$. Left at the end of the acceleration phase after one chord length of travel $\left(\delta^{*}=1\right)$; Right after $\delta^{*}=1.5$. For the complete evolution throughout the revolving motion, see the supplementary animation (Online Resource 2) 
views showing the increasing tip deflection with decreasing flexural stiffness. For each wing, a similar vortex structure can be observed, where an LEV, an initial coherent starting TEV that is followed by a number of smaller scale TEVs, a TV, and an RV are present in the flow fields. However, a more coherent vortex system (i.e., with less fragmentation of the structures) is observed for the flexible wings with the most significant difference occurring for the RV. In consequence of the chordwise deflection of the flexible wings, the $\mathrm{TV}$ and the starting vortex system is more elongated in the streamwise direction. As a result, the vortical structures in the wake are confined to a significantly smaller region. At $\delta^{*}=1.5$, the formation of the arch-shaped LEV structure at the outboard locations of the three wings takes place similarly independent from the structural deformation. It can be argued that the LEV stays closer to the wing surface in the case of the flexible wings, which will be further analyzed in Sect. 4.2.3. Another prominent difference between the flow fields of the wings is observed regarding the helical density, which is indicative of an outboard spanwise vorticity flux along the axis of the LEV, as also reported by Percin and van Oudheusden (2015a). It can be observed that, for the flexible wings, the regions of positive helicity associated with the LEV become more coherent, suggesting higher levels of spanwise vorticity transport for the flexible wings. Especially, in the earlier phases of the revolving motion $\left(\delta^{*}<1.5\right)$, before the onset of vortex burst, high positive helicity values are seen for the flexible wings. This observation is in agreement with the increased levels of vorticity flux $\left(q_{L E V}^{*}\right)$ along the wing span as given in Fig. 14, which is further investigated in Sect. 4.2.3.

\subsubsection{Spanwise vorticity and flow}

In Fig. 12, contours of spanwise vorticity are given in chordwise-oriented planes along the span and at the mid-span $(r / R=0.5)$ and the reference plane position $(r / R=0.75)$ for $\delta^{*}=1.0,1.5,2$, and 4 . At the start of the revolving motion, the spanwise-oriented vorticity contours display rather similar quasi-two-dimensional characteristics for the different wings (this is also evident from the relatively constant LEV vorticity flux $q_{L E V}^{*}$ at $\delta^{*}=1$, see Fig. 14 in Sect. 4.2.3). As the motion progresses, these coherent structures start to develop three-dimensional features with the region of the positive LEV vorticity enlarging in the spanwise direction. The positive vorticity contour emanating from the leading edge interacts with the negative vorticity that is generated between the LEV and the wing, which leads to the formation of discrete pockets of both negative and positive vorticity at about $\delta^{*}=1.5$ and 2 . The cross-sectional area containing the entrained vorticity of the LEV expands during these time steps and breakdown of the LEV particularly at the outer span locations occurs, which can also be evidenced from the three-dimensional vortex structures shown in Fig. 10 for the rigid wing case. At $\delta^{*}=4$, the LEV extends over the complete suction side of the wings. It also interacts with the negative vorticity emanating from the trailing edge as clearly visible at the $r / R=0.75$ spanwise location. This mechanism has been claimed to be the limiting factor in the force generation by Garmann and Visbal (2014). It is also observed that the overall cross-sectional area occupied by the recirculating flow containing the entrained vorticity is significantly smaller with decreasing flexural stiffness. This is especially pronounced within the bubble-like structure $(r / R>0.5)$.

In Fig. 13, the spanwise velocity (i.e., the velocity component parallel to the leading edge) is given in chordwise-oriented planes along the span, as well as at the mid-span position $(r / R=0.5)$ and at the reference position $(r / R=0.75)$ in the inertial reference frame for $\delta^{*}=1.5$ and 4 . It is clear that the wing flexibility does not have a significant impact on the overall topology of the spanwise flow pattern apart from small variations in the coherency of the structures. At $\delta^{*}=1.5$, an outboard-directed spanwise flow (positive $U_{z}$ ), at a position which overlaps with the LEV, is present along most of the wing span. Close to the wing tip, an inboarddirected spanwise flow occurs in relation to the TV. However, the effect of the TV weakens substantially as the motion progresses and the TV loses its coherency, which is evident from the outward-directed flow pattern at the wing tip at $\delta^{*}=4$. The spanwise position of the TV is comparable for the different flexibilities. In correlation with the behavior of the LEV, the positive spanwise flow patterns, which are likely to stem from centrifugal effects, also cover the complete suction side indicating the presence of a considerable amount of vorticity flux toward the wing tip. At this time step, the shear layer emanating from the trailing edge is visible as the interface between spanwise velocity above the shear layer and negligible spanwise velocity below it. For decreasing flexural stiffness, the interface of the shear layer is smoother with less Kelvin-Helmholtz like instability, which is associated with the more continuous shedding of TEVs (see Fig. 12).

\subsubsection{Spanwise wing characteristics in terms of the LEV properties}

The spanwise distribution of the LEV circulation, LEV vorticity flux, and LEV centroid position for $\delta^{*}=1,1.5$, and 4 is given in Fig. 14. The occurrence of jumps is visible in the overlap regions of the measurement volumes due to imperfect data match between the adjacent volumes. However, these jumps are not prohibitive in assessing the behavior of the different parameters and the impact of wing flexibility.

In conjunction with the temporal evolution of the threedimensional flow fields, until the end of the acceleration period $\left(\delta^{*}=1\right)$, all wings display very similar characteristics 


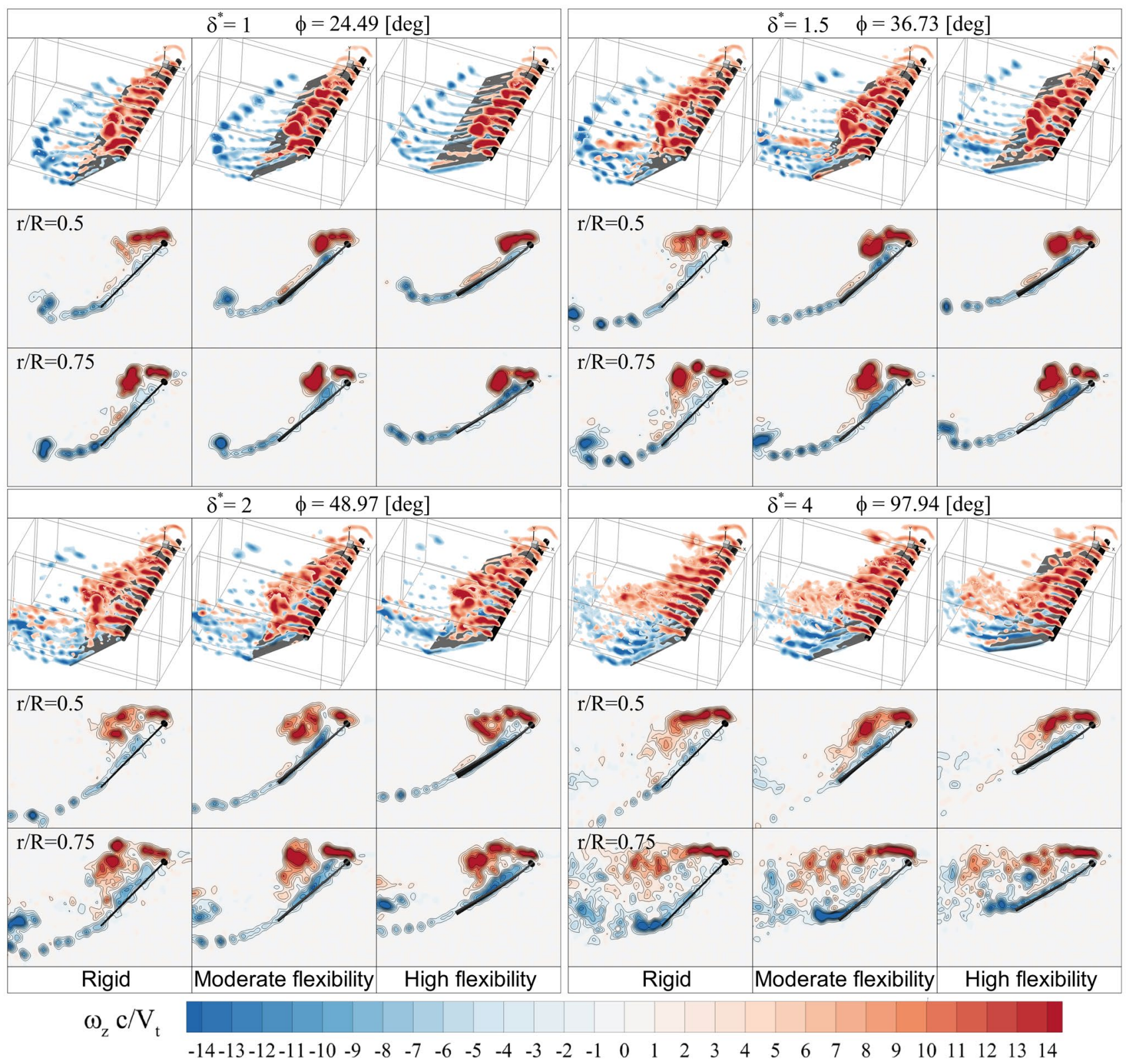

Fig. 12 Spanwise vorticity contours $\left(\omega_{z} c / V_{t}\right)$. Vorticity contours along the span, at $r / R=0.5$ and at $r / R=0.75$, for $\delta^{*}=1,1.5,2$, and 4 . For the complete evolution throughout the revolving motion; see the supplementary animation (Online Resource 3 )

in terms of the LEV circulation and its centroid position. Subsequently, at about $\delta^{*}>1.5$, a transition occurs which correlates with the onset of vortex burst. Finally, at around $\delta^{*}=4$, the spanwise distribution is settled and approximately steady-state conditions are reached.

The LEV circulation at $\delta^{*}=1$ is similar for the different wings and shows a linear increase of circulation with spanwise position until $r / R=0.9$, which is associated with the increase in rotational velocity due to the curvilinear nature of the motion, after which it decreases to zero at the tip. At steady-state conditions $\left(\delta^{*}=4\right)$, the circulation inboard of mid-span $(r / R<0.5)$ is similar for the different wings, while outboard of mid-span, the circulation is decreased significantly with decreasing flexural stiffness. For $\delta^{*}<1$, there is a little spanwise transport of vorticity within the $\operatorname{LEV}\left(q_{L E V}^{*}\right)$ and the flow behaves as nearly two-dimensional (see Fig. 12). Subsequently, there is a very strong increase of positive spanwise vorticity flux, followed by a transition after which a relatively constant positive level of spanwise advection of vorticity within the LEV is established at $\delta^{*}=4$. Furthermore, it can be noted that the spanwise advection of vorticity within the LEV is stronger for the flexible wings compared to the rigid wing. The observations are in agreement with the 


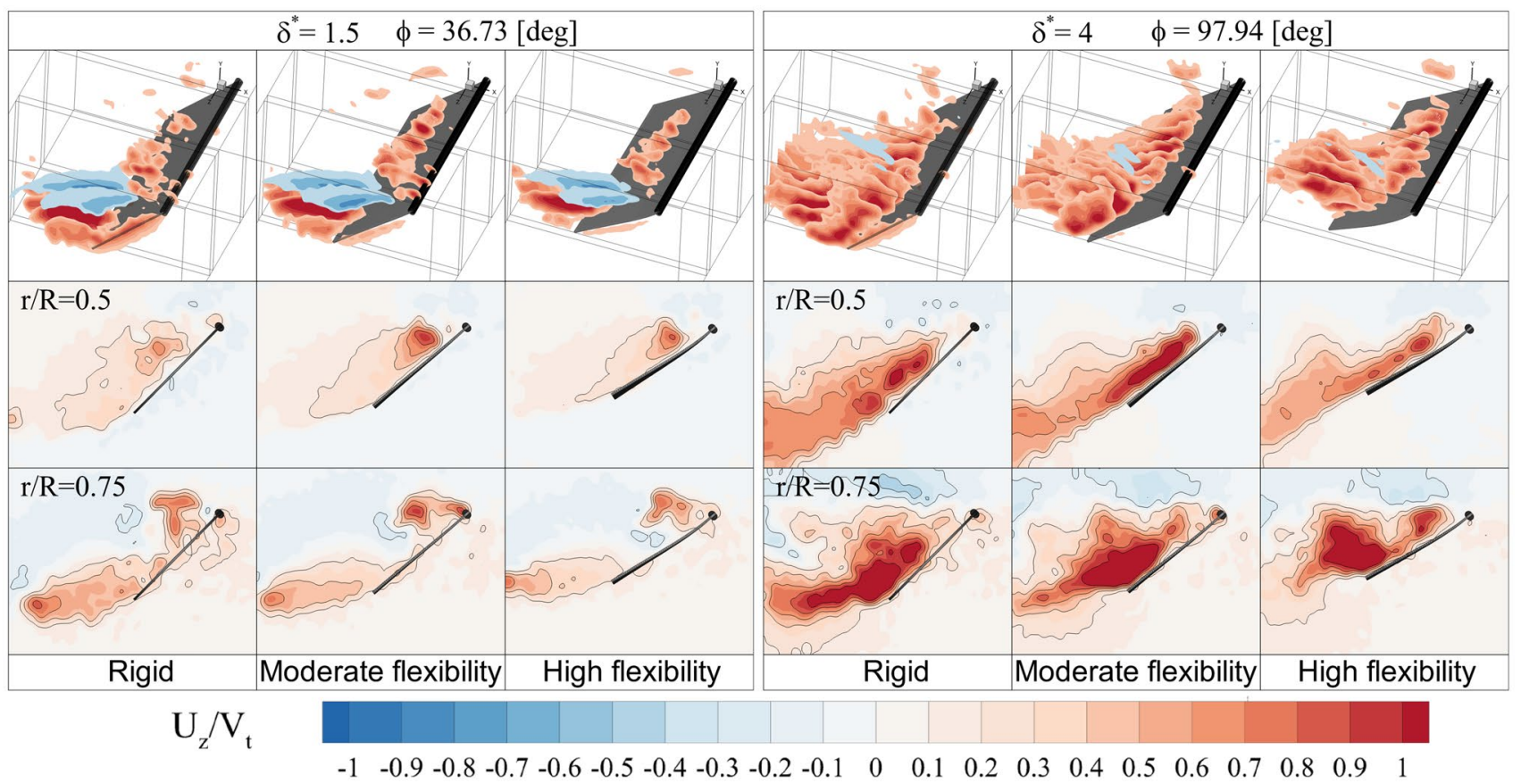

Fig. 13 Spanwise velocity $\left(U_{z} / V_{t}\right)$ for $\delta^{*}=1.5$ and 4. Top Spanwise velocity along the span. Middle, bottom Spanwise velocity contours at $r / R=0.5$ and at $r / R=0.75$. For the complete evolution throughout the revolving motion; see the supplementary animation (Online Resource 4)

increased levels of helical density of the LEV core, which is better aligned in the spanwise direction for the flexible wings, as shown in Fig. 11.

From the LEV centroid position, the core of the LEV arch-shaped bubble-like structure can be seen. It is found that, for increasing flexibilities, the core of the LEV lifts off less from the wing surface, which is most pronounced outboard of mid-span $(r / R>0.5)$ at $\delta^{*}=4$. The aft tilt of the LEV is approximately similar for the different flexibilities, except near the tip $(r / R>0.6)$ at $\delta^{*}=4$, which corresponds with the bubble-like structure. This structure does not extend downstream in the case of deforming wings as much as for the rigid wing.

\subsubsection{Conclusion}

A similar vortex system, comprising LEV, TV, RV, and starting TEV components, is observed in all the cases. For decreasing flexural stiffness, the coherency of this vortex system is increased. In the earlier phases of the revolving motion, the LEV structure of the flexible wings shows higher helical density values that match with increased outboard spanwise vorticity flux along the axis of the LEV, which may be associated with the prolonged retention of the LEV. At later phases of the revolving motion, vortex breakdown occurs for all wings.

\subsection{Force production mechanisms}

In the discussion of the force production mechanisms, first, the three-dimensional pressure fields are analyzed in relation to the coherent vortical structures. Next, the spanwise variation of the sectional forces is discussed, and connected to the LEV properties.

\subsubsection{Three-dimensional pressure fields}

The pressure fields at two time instants $\left(\delta^{*}=1.5\right.$ and 4$)$ are presented in Fig. 15 by means of positive and negative isosurfaces, superimposed on isosurfaces of the $Q$-criterion, to highlight the relation between the pressure fields and the vortical structures. It is clear that the presence of the LEV results in the formation of a considerable suction region, which enlarges and deforms in accordance with the development of the LEV. The suction region further extends over the TV and the starting vortex, albeit that pressure features corresponding to the small-scale vortical structures (i.e., secondary TEVs) are not resolved due to the limited spatial resolution and smoothing nature of the Poisson solver integration scheme. The characteristics of the pressure fields differ distinctly between the different wing cases particularly in size and in position with respect to the wing surface. The high-pressure region on the pressure side of the wing is mainly located outboard of the mid-span location 


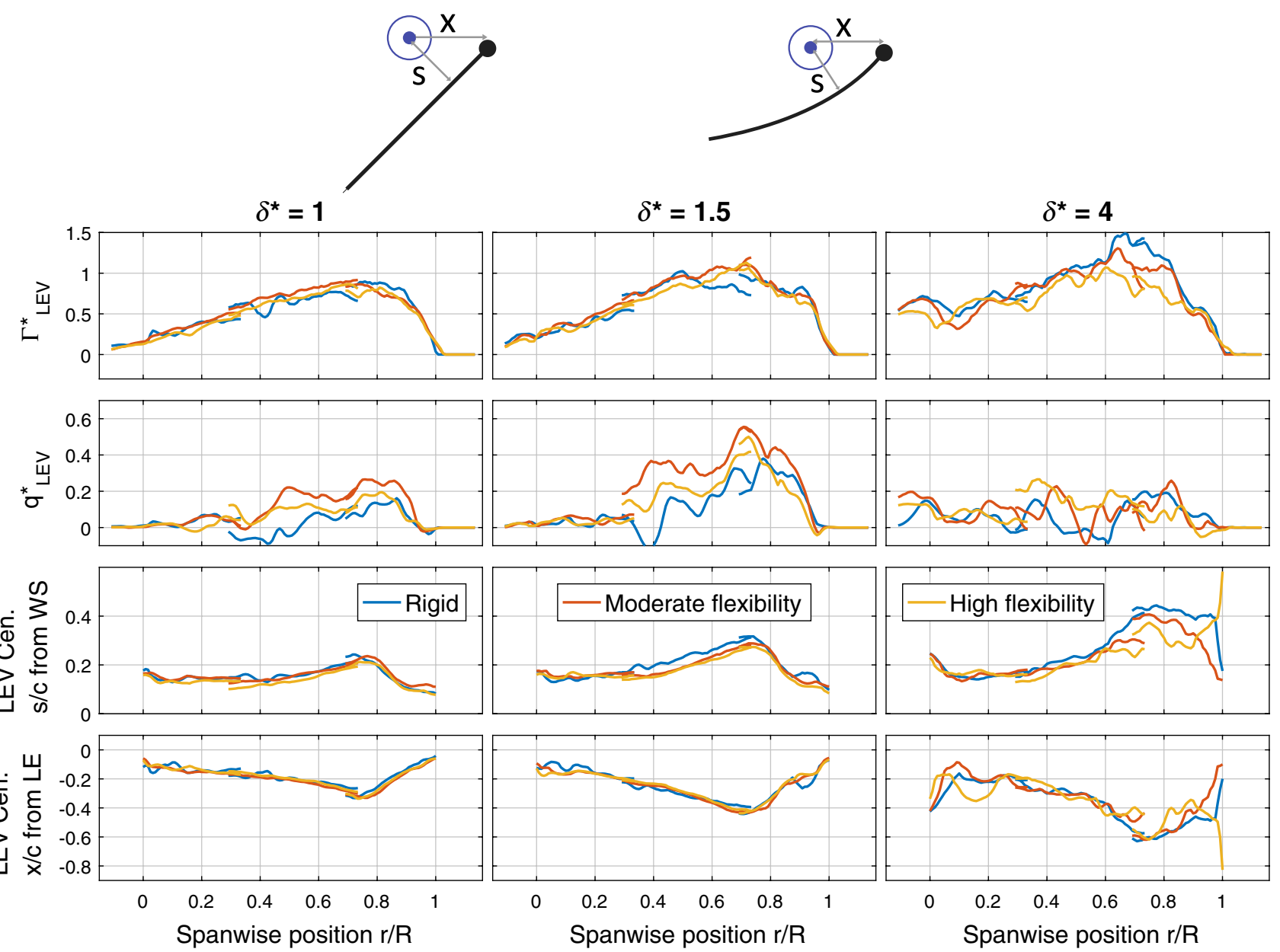

Fig. 14 Spanwise characteristics for $\delta^{*}=1,1.5$, and 4. From the top to bottom row, respectively: LEV circulation $\left(\Gamma_{L E V}^{*}\right)$, LEV vorticity flux $\left(q_{L E V}^{*}\right), \mathrm{LEV}$ centroid chord distance $(s / c)$ normal to wing surface (WS), LEV centroid chord distance in the $x$-direction $(x / c)$ from lead-

$(r / R>0.5)$ and it clearly reduces in size with decreasing flexural stiffness as is especially evident in the side views.

For a more detailed visualization of the low-pressure regions, which are associated with the vortex system, pressure contours are plotted for the different wings in chordwise-oriented planes along the span, and at $50 \%$ and $75 \%$ span locations for $\delta^{*}=1.5$ and 4 in Fig. 16. This shows that the LEV pressure centroid location is similar for the different wings in absolute sense which is verified by means of its centroid position as function of spanwise position (see Sect. 4.3.2 Fig. 17). In correlation with the position of the LEV, the suction peak is also located closer to the wing surface for decreasing flexural stiffness, which is advantageous for the force production. Moreover, the size of the suction region accompanying the LEV reduces for decreasing flexural stiffness which is particularly evident at the outboard spanwise locations at the later stages of the ing edge (LE) as a function of spanwise position along the span $(r)$ non-dimensionalized with the span length $(R)$. The sectional control volumes have a spanwise thickness of $\mathrm{d} z=7$ vectors and an overlap of 6 vectors

motion. Also considering the reduction in the size of the high-pressure region, the resultant net pressure difference between the pressure and suction side of the airfoil is significantly decreased with decreasing flexural stiffness. Despite this decrease in the resultant force, the corresponding lift generation remains relatively high because of the increased alignment of the force vector with the direction of the lift caused by the wing deformation (see Figs. 4 and 5). These observations suggest a reduction of the total net force and an increase of the lift-to-drag ratio, which agrees well with the direct force measurements, as depicted in Fig. 7.

\subsubsection{Spanwise variation of forces}

The spanwise variation of forces is studied by analyzing the LEV pressure centroid position and the sectional lift and drag coefficients for $\delta^{*}=1,1.5$, and 4 as given in Fig. 17 . 


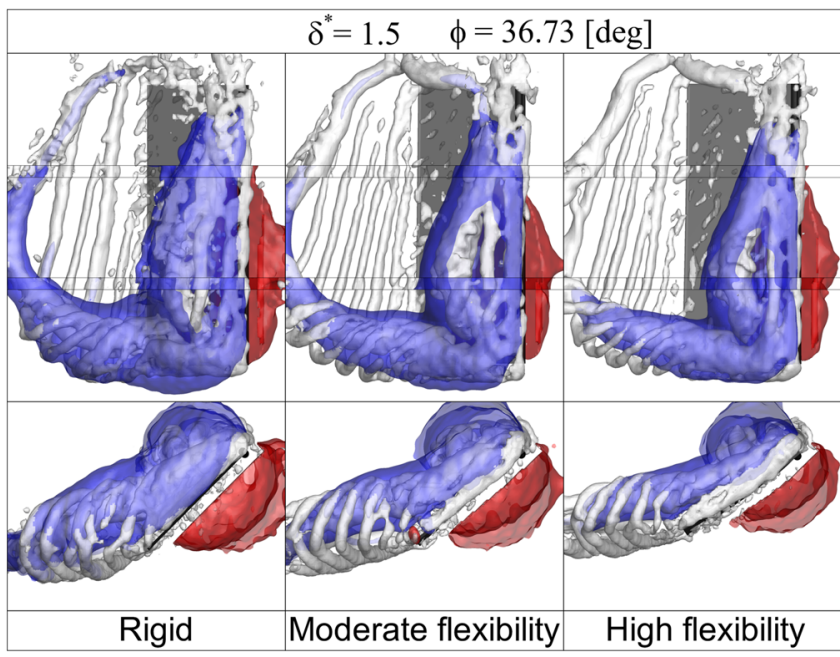

Fig. 15 Vortical structures and reconstructed pressure fields for $\delta^{*}=1.5$ and 4. Isosurfaces of $Q$-criterion: $Q /\left(V_{t} / c\right)^{2}=3$ (White). Isosurfaces of reconstructed pressure field: $p=-13 \mathrm{~Pa}$ (Blue) and

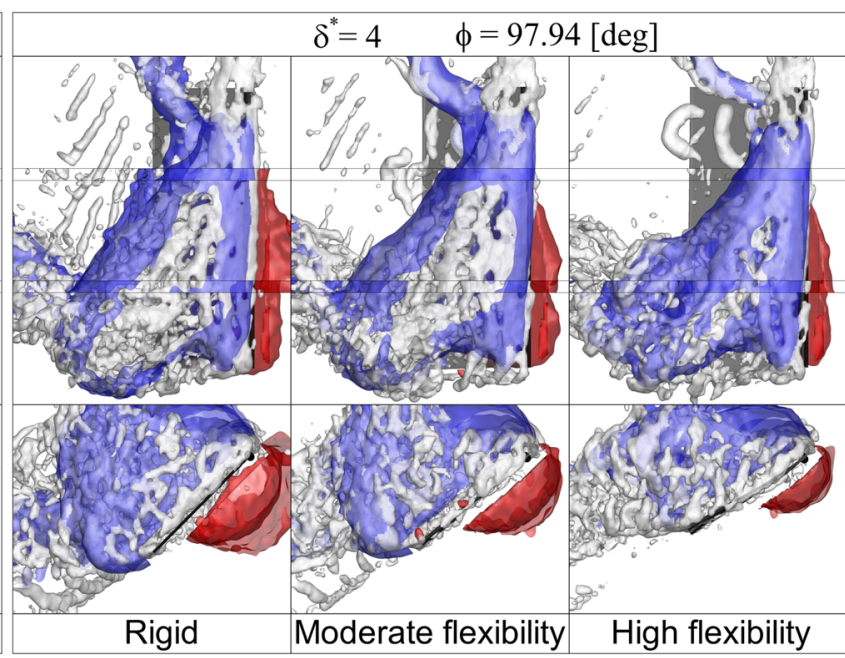

$p=6 \mathrm{~Pa}$ (Red). For the complete evolution throughout the revolving motion, see the supplementary animation (Online Resource 5)

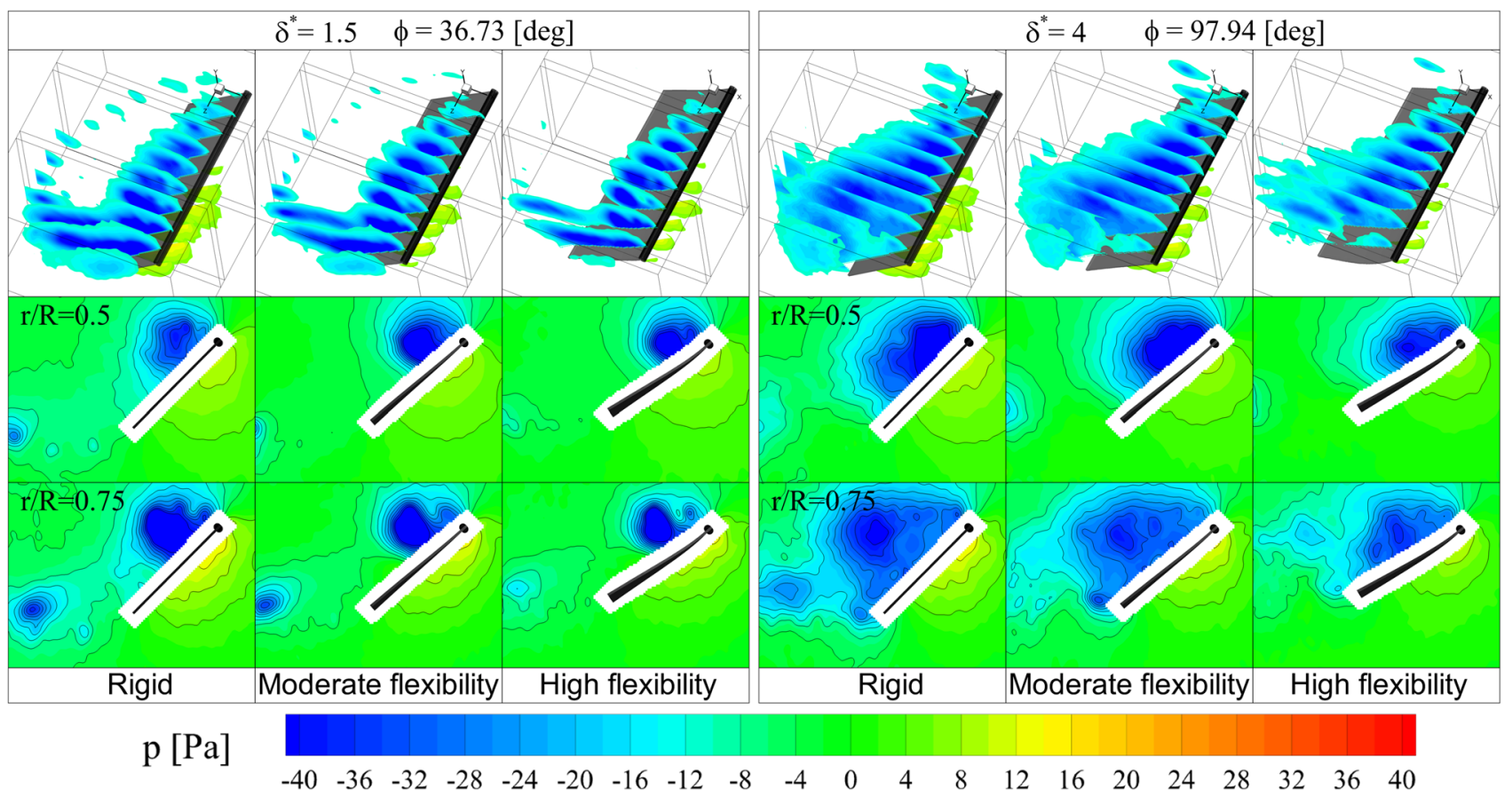

Fig. 16 Pressure contours $(p)$ for $\delta^{*}=1.5$ and 4. Top Pressure contours along the span. Middle, Bottom Pressure contours at $r / R=0.5$ and at $r / R=0.75$. For the complete evolution throughout the revolving motion, see the supplementary animation (Online Resource 6)

First, as expected, the LEV pressure centroid has a high correlation with the LEV centroid (compare Fig. 14) for the different flexibilities with the most pronounced difference near the tip at $\delta^{*}=4$. This agreement between the pressure and LEV centroid positions is in accordance with Figs. 15 and 16, in which the low-pressure region correlates well with the LEV structure.
From the spanwise LEV pressure centroid position (Fig. 17, first row), it is observed that the suction peak is located significantly closer to the wing surface for decreasing flexural stiffness, such that the force production of the flexible wings is relatively high. This is most pronounced outboard of mid-span $(r / R>0.5)$ at $\delta^{*}=4$ and correlates 


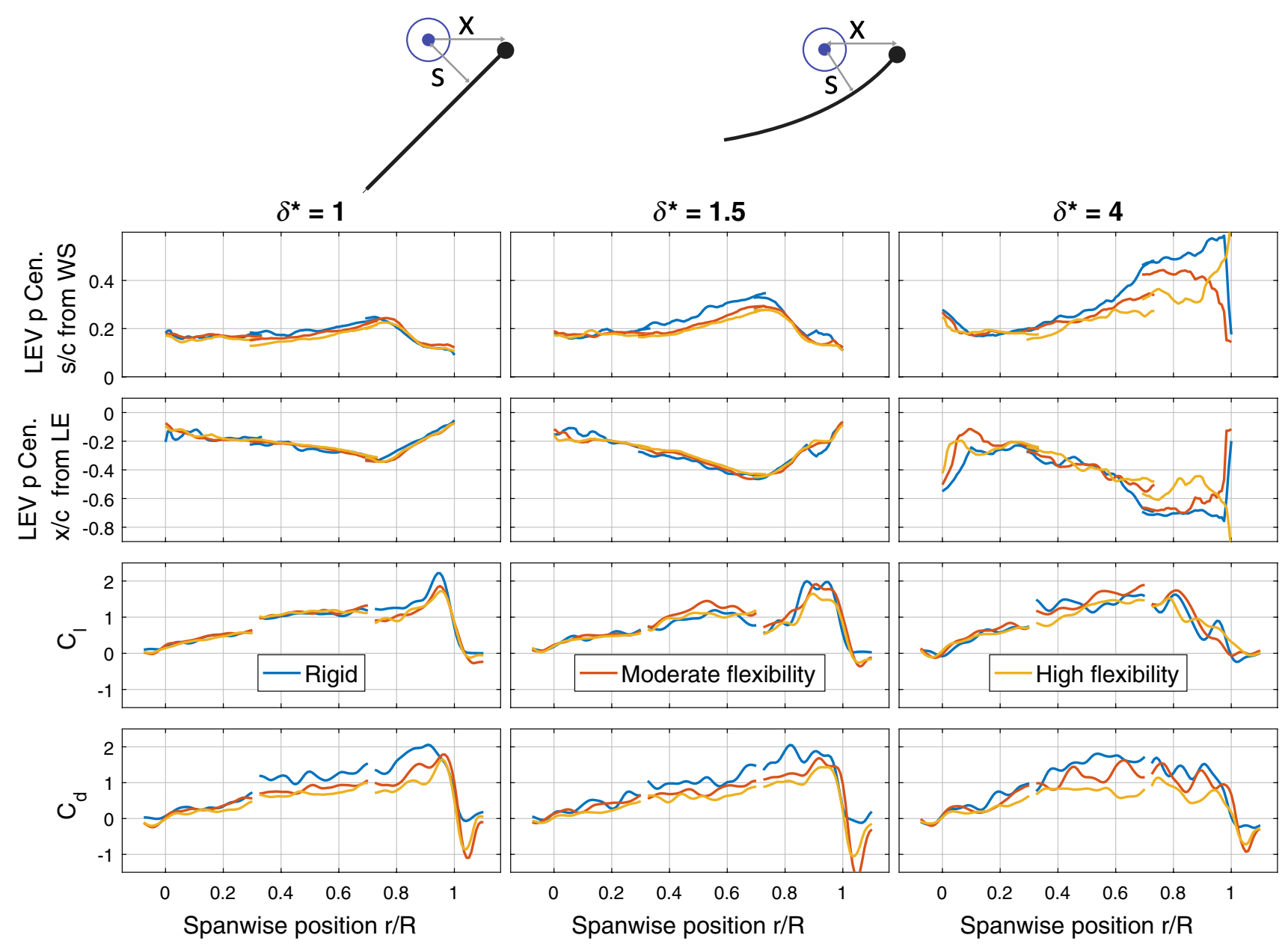

Fig. 17 Spanwise characteristics for $\delta^{*}=1,1.5$ and 4. From the top to bottom row, respectively: LEV pressure centroid chord distance $(s / c)$ normal to wing surface (WS), LEV pressure centroid chord distance in the $x$-direction $(x / c)$ from leading edge (LE), sectional lift

with the observations of the spanwise pressure fields, as depicted in Fig. 16.

The sectional lift and drag at the end of the acceleration $\left(\delta^{*}=1\right)$ show a linear increase with spanwise position until approximately $r / R=0.9$ in line with the increase in rotational velocity and LEV circulation. While the sectional lift for the different wings is comparable, a significant decrease in sectional drag can be observed with decreasing flexural stiffness. This decrease in drag is mainly found at the outboard wing locations, while, inboard of $r / R=0.3$, the sectional drag is approximately similar for the different wings. The regions of reduced drag correspond to the spanwise locations where the LEV is significantly expanded generating a considerable suction region that is responsible for a relatively high net sectional force. In addition, towards the wing tip, the flexible wings deflect more (see Fig. 5). As coefficient $\left(C_{l}\right)$, and sectional drag coefficient $\left(C_{d}\right)$ as a function of spanwise position along the span $(r)$ non-dimensionalized with the span length $(R)$. The sectional control volumes have a spanwise thickness of $d z=7$ vectors and an overlap of six vectors

such, this increased wing deflection towards the wing tip together with its relatively high sectional force results in a relatively high lift at the outboard wing locations, while the drag is significantly suppressed.

The corresponding spanwise centroids of the lift and drag are located at approximately $70 \%$ of the span for all the wings and throughout the complete motion; see Fig. 18. This spanwise position may be seen to represent the characteristic spanwise section at the considered Rossby number, and the observation that it is relatively independent of chordwise wing deformation may simplify the modeling of flexible wings in the context of flapping-wing flight. The spanwise characteristics indicate that, at this span position, the LEV circulation is approximately highest (see Fig. 14) and the suction peak is located furthest downstream with the largest distance from the airfoil. 

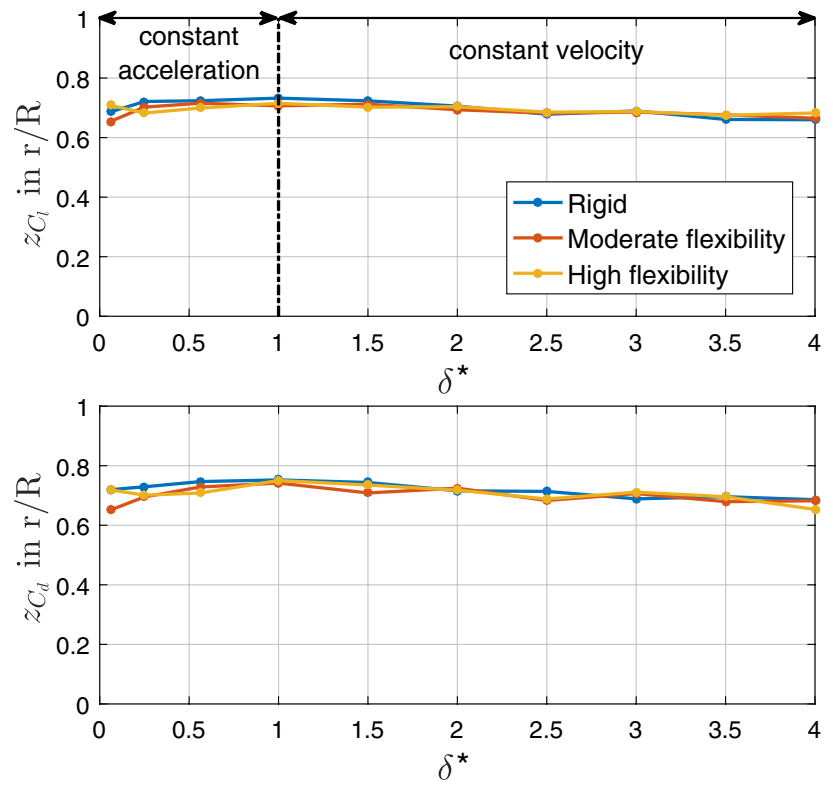

Fig. 18 Temporal evolution of the spanwise centroid position for the lift (top) and drag (bottom)

\subsubsection{Conclusion}

The LEV generates a considerable suction region and plays a prominent role in the force production mechanism of the revolving wings. By combining the pressure, flow field, and balance data observations, the relatively high lift and the significantly reduced drag for the flexible wings are explained: as the low-pressure region accompanying the LEV becomes smaller with increasing flexibility, the total force acting on the wing is reduced, but it is also tilted more towards the lift direction due to the wing deformation. As a consequence, the lift component remains relatively high, also because the suction peak is located closer to the wing surface. Simultaneously, the drag is significantly suppressed for increasing flexibility. The corresponding spanwise centroids of lift and drag are located at approximately $70 \%$ of the span for all the wings throughout the complete revolving motion.

\subsection{On the relation between pressure gradient and LEV behavior}

The specific focus of this section is on the evolution of the (spanwise) pressure gradient in connection to the development and bursting behavior of the LEV. First, the rigid wing is investigated in detail, after which a comparison between the different flexible wings is made.

The pressure gradient is calculated as the numerical spatial gradient of the reconstructed pressure field and can be related to the fluid material acceleration in view of the momentum equation, see Eq. 2. The process of vortex breakdown has been found to correlate well with the formation of a positive spanwise pressure gradient over the wing (Garmann and Visbal 2014; Jardin and David 2014), which is indicative for a negative spanwise acceleration (inboard) of a particle within the flow field. As the outboard moving particles experience an adverse pressure gradient imposing an inboard acceleration, the spanwise advection of vorticity is reduced and vorticity is accumulated in a given plane, which ultimately leads to the burst of the vortical structure.

In Fig. 19, the temporal evolution of the advection of vorticity and spanwise pressure gradient is given for the rigid wing, which illustrates the gradual development of the process of breakdown. Early in the revolving motion, starting around $\delta^{*}=0.5625$, a region of negative spanwise pressure gradient is formed at the suction side of the wing. This is associated with a promotion of the outboard spanwise advection of vorticity $\left(\omega_{z} U_{z} c / V_{t}^{2}\right)$ and balances the production of vorticity at the leading edge, such that a stable LEV is maintained. The negative pressure gradient region that has formed for $\delta^{*}<1.5$ correlates well with the location of the LEV core (identified by the $Q$-criterion) and its strong levels of vorticity flux density (identified by its high helical density values). This agreement is also evident from spanwise plane at the mid-span position $(r / R=0.5)$ where the negative pressure gradient region (see the bottom row of Fig. 19) matches with the region of positive spanwise vorticity flux (see the second row of Fig. 19). Subsequently, for $\delta^{*}>1.5$, around the revolving phases when the burst of the LEV initiates, also a region of positive pressure gradient is formed around mid-span near the leading edge which correlates with the formation of negative spanwise vorticity flux. As a result, the spanwise advection of vorticity is decreased and vorticity is accumulated which ultimately leads to the burst of the LEV. A further region of positive pressure gradient is formed near the tip of the wing above the region of negative pressure gradient. Subsequently, the region of positive pressure gradient around mid-span starts expanding. Simultaneously, the region of positive pressure gradient near the tip connects with the region of positive pressure gradient over the wing. Both regions start merging until a coherent region of positive and negative pressure gradient is formed at, respectively, the outboard and inboard part of the wing for steady-state conditions $\left(\delta^{*}=4\right)$.

Next, in Fig. 20, the spanwise pressure gradients of the different wing models are compared. The general topological distribution of the spanwise pressure gradient is very similar with little dependence on the wing flexibility. Nevertheless, some small differences are observed. First, at mid-span $(r / R=0.5)$ higher negative spanwise pressure gradient values are observed for the two flexible wings. These increased negative spanwise pressure gradient values around mid-span are in agreement with the increased levels of helical density of the LEV core (see Fig. 11) and the increased vorticity 


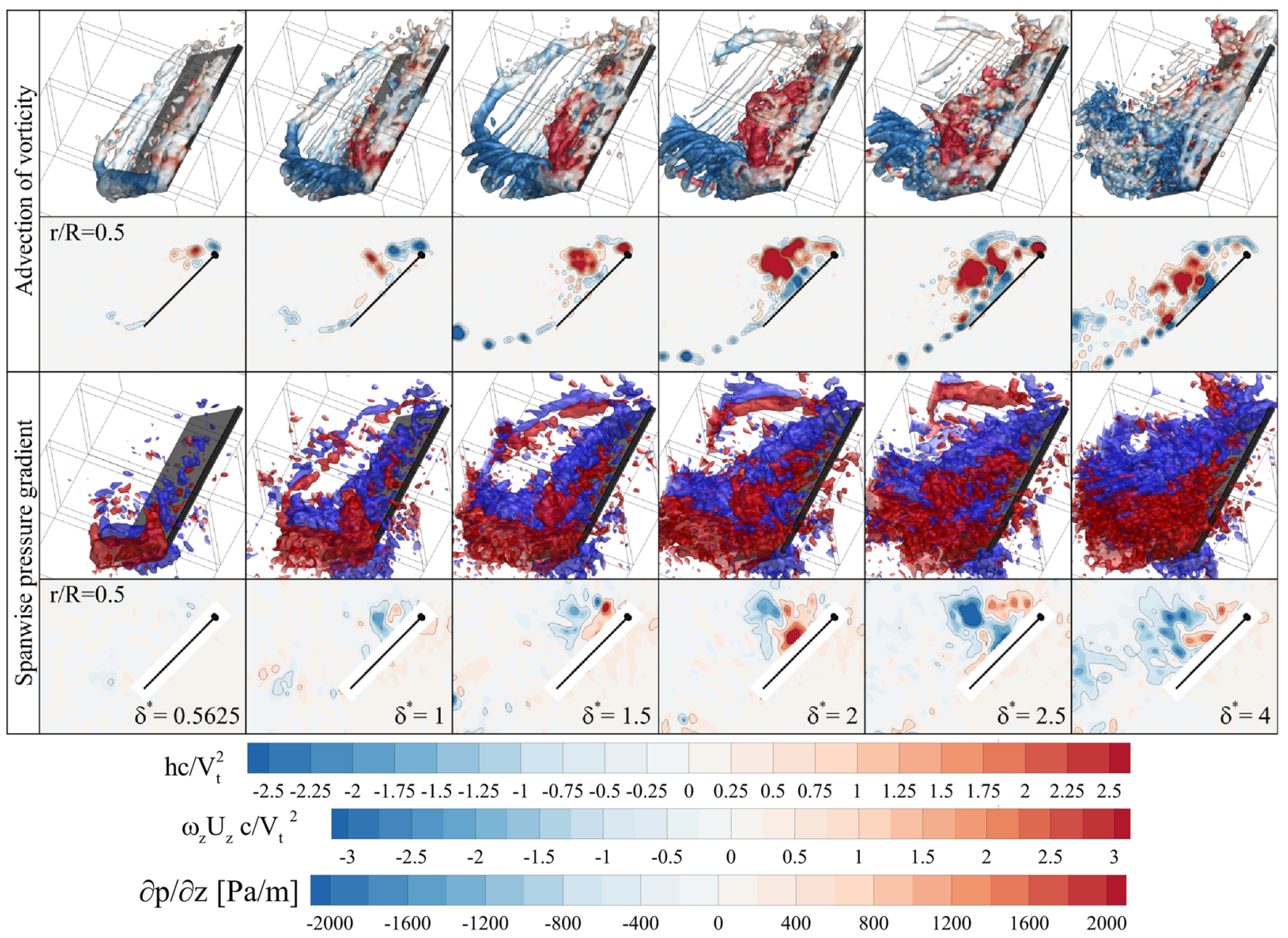

Fig. 19 Temporal evolution of advection of vorticity and spanwise pressure gradient for the rigid wing. Top row Isosurfaces of $Q$-criterion $Q /\left(V_{t} / c\right)^{2}=3$ (White) colored by helical density $\left(h c / V_{t}^{2}\right)$. Second row Spanwise vorticity flux $\left(\omega_{z} U_{z} c / V_{t}^{2}\right)$ at $r / R=0.5$.
Bottom two rows Isosurfaces of spanwise pressure gradient: $\partial p / \partial z=-500 \mathrm{~Pa} / \mathrm{m}$ (Blue) and $\partial p / \partial z=500 \mathrm{~Pa} / \mathrm{m}($ Red) and $\partial p / \partial z$ contours at $r / R=0.5$

spanwise flow. However, outside the vortex core, the centrifugal force was found to be equally important in the formation of spanwise flow. The dominant role of the pressure gradient in generating the outboard spanwise flow in the core of the LEV was also observed in Jardin and David (2014). In addition, (Jardin 2017) showed that the Coriolis effect increases the spanwise flow in both the core and behind the LEV by enhancing pressure gradients. This spanwise flow advects vorticity along the span which stabilizes the LEV (Ellington et al. 1996). These observations are in agreement with (Lentink and Dickinson 2009) who concluded that the pressure gradient force can explain the spanwise flow in the LEV core, whereas centrifugal pumping can explain the spanwise flow outside the LEV core. The current experimental study supports these findings; in the sense that the LEV core including its strong levels of spanwise transport of vorticity that are driven by an outboard spanwise velocity seems to coincide well with the region of negative spanwise the LEV and it is responsible in the formation of outboard 


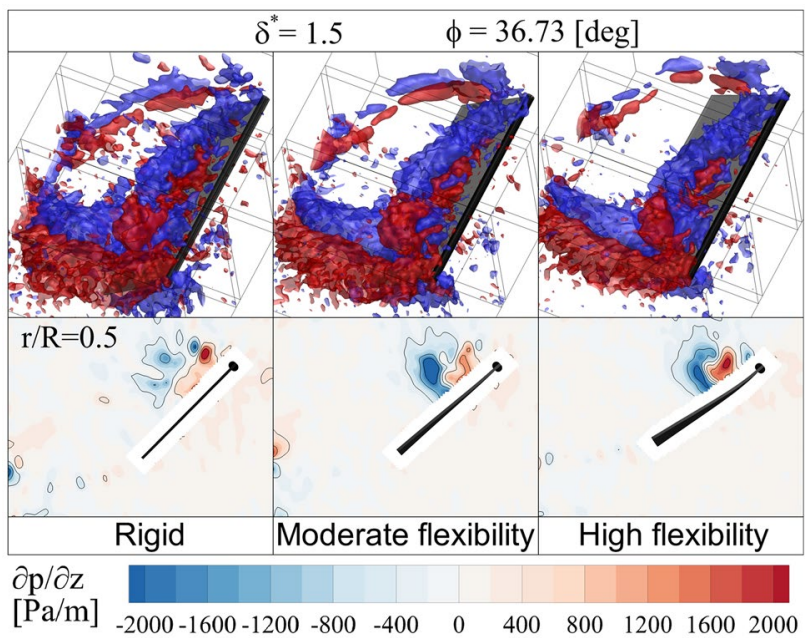

Fig. 20 Comparison of spanwise pressure gradient at $\delta^{*}=1.5$. Top Isosurfaces of spanwise pressure gradient: $\partial p / \partial z=-500 \mathrm{~Pa} / \mathrm{m}$ (Blue) and $\partial p / \partial z=500 \mathrm{~Pa} / \mathrm{m}$ (Red). Bottom $\partial p / \partial z$ contours at $r / R=0.5$. For the complete evolution throughout the revolving motion, see the supplementary animation (Online Resource 7)

pressure gradient. Downstream of the LEV core, a relatively large region of spanwise velocity is observed (see Fig. 13, $r / R=0.5, \delta^{*}=1.5$ ), while the pressure gradient is negligible. These spanwise velocities are likely to result from the centrifugal effects.

Concluding, a good agreement between the process of vortex breakdown and the formation of a positive spanwise pressure gradient is observed. In addition, before the burst of the LEV, increased levels of negative spanwise pressure gradient are seen to occur around mid-span for the flexible wings. This is in agreement with the increased spanwise advection of vorticity and contributes to the prolonged coherence and retention of the LEV for the flexible wings.

\section{Conclusion}

The flow fields and fluid-dynamic loads generated by revolving low-aspect-ratio flat-plate wings with different degrees of chordwise flexibility have been experimentally studied using tomographic-PIV and simultaneous force measurements. Three different wings were tested in the experiments to investigate the influence of wing flexibility: a rigid wing, a moderately flexible wing, and a highly flexible wing. At the low Reynolds number regime considered in the present experiments, the flow field is highly repeatable, notably for the initial development phase of the flow, which allows obtaining temporal information from phase-locked measurements. The pressure field and fluid-dynamic loads have been reconstructed successfully from the ensemble-averaged phase-locked tomographic-PIV measurements for a volumecontaining the entire wing.

The force-balance measurements reveal that, during the acceleration phase, the build-up of lift is similar for the different wings, while the build-up of drag is significantly lower with decreasing flexural stiffness (see Fig. 7). At steady-state conditions, the net resultant force generated is decreased significantly with decreasing flexural stiffness. However, this does not adversely affect the lift generation, such that the lift for the rigid and the moderate flexible wings is very similar, while it is only slightly (about 7\%) smaller for the high flexible wing. On the other hand, the drag decreases monotonically with decreasing flexural stiffness, up to $36 \%$ for the most flexible wing. As a result, the most flexible wing has an increased lift-to-drag ratio of approximately $45 \%$ (see Fig. 7d). It is further found that, for the chordwise-flexible wing configurations considered in this study, the lift-to-drag ratio at the steady-state conditions correlates well with the geometric angle of attack. Moreover, it is shown that a rigid wing with a geometric angle of attack identical to that of the deformed chordwise-flexible wing generates similar lift and drag, which suggests that, at steady-state conditions, the geometric angle of attack is representative for the resultant force (see Table 2). This could potentially simplify the modeling of flexible wings, since the fluid-dynamic forces can be approximated based on an equivalent rigid wing model.

A similar vortex system, comprising LEV, TV, RV, and starting TEV components, is observed in all the cases. For decreasing flexural stiffness, the coherency of this vortex system is increased. In the earlier phases of the revolving motion, the LEV structure of the flexible wings shows higher helical density values (see Fig. 11) that match with increased outboard spanwise vorticity flux along the axis of the LEV (see Fig. 14), which may be associated with the prolonged coherence and retention of the LEV. At later phases of the revolving motion, vortex breakdown occurs for all wings.

The pressure field and loads have been reconstructed from the phase-resolved volumetric velocity data, employing the non-inertial moving reference frame of the rotating wing. The vortex system structures encompass a low-pressure region which has a high correlation with the vortical structures, as identified by the $Q$-criterion (see Fig. 15).

Due to the same predefined local angle of attack of $45^{\circ}$ at the leading edge for the different wings, the circulation of the flexible wings is relatively high and over a large extent of the span and the revolving motion, similar to that of the rigid wing. The LEV pressure centroid (see Fig. 17) is in agreement with the LEV centroid (see Fig. 14) and is comparable for the different wings in absolute sense. However, due to the deflection of the flexible wings, the LEV pressure centroid is located closer to the wing surface which is most pronounced at the outboard wing locations. These observations, based on the flow field data, can be put in 
relation to the results from the force-balance measurements. As the low-pressure region accompanying the LEV becomes smaller with increasing flexibility, the total force acting on the wing is reduced, but it is also tilted more towards the lift direction due to the wing deformation. As a consequence, the lift component remains relatively high, also because the suction peak is located closer to the wing surface. Simultaneously, the drag is significantly suppressed for increasing flexibility. While the sectional lift along the full span is comparable for the different wings, the sectional drag is significantly reduced for increasing flexibility at the outboard wing locations (see Fig. 17). These locations of reduced drag correspond to the spanwise locations in which the LEV is significantly expanded. The corresponding spanwise centroids of lift and drag are located at approximately $70 \%$ of the span for all wings throughout the complete revolving motion (see Fig. 18).

An agreement between the process of vortex breakdown and the formation of a positive pressure gradient is observed (see Fig. 19). As the outboard moving fluid particles experience a positive adverse pressure gradient imposing an inboard acceleration, the spanwise advection of vorticity is inhibited and vorticity is accumulated, which can eventually lead to the burst of the vortical structure. Early in the revolving motion, a negative spanwise pressure gradient is formed at the suction side of the wing which drives the high spanwise transport of vorticity of the LEV structure. However, at the onset of vortex breakdown, around mid-span, a region of positive spanwise pressure gradient is formed near the leading edge. Subsequently, this region starts expanding, and at steady-state conditions, a region of positive and negative pressure gradient is present at, respectively, the outboard and inboard parts of the wing. For the flexible wings, increased values of negative spanwise pressure gradient are observed around mid-span (see Fig. 20) before the burst of the LEV. This is in agreement with the increased spanwise advection of vorticity (see Fig. 14) and contributes to the prolonged coherence and retention of the LEV for the flexible wings.

Open Access This article is distributed under the terms of the Creative Commons Attribution 4.0 International License (http://creativeco mmons.org/licenses/by/4.0/), which permits unrestricted use, distribution, and reproduction in any medium, provided you give appropriate credit to the original author(s) and the source, provide a link to the Creative Commons license, and indicate if changes were made.

\section{References}

Anderson JD (2011) Fundamentals of aerodynamics, 5th edn. Anderson series. McGraw-Hill, New York

Beals N, Jones AR (2015) Lift production by a passively flexible rotating wing. AIAA J 53(10):2995-3005. https://doi.org/10.2514/1. J053863
Birch JM, Dickson WB, Dickinson MH (2004) Force production and flow structure of the leading edge vortex on flapping wings at high and low reynolds numbers. J Exp Biol 207(7):1063-1072. https:// doi.org/10.1242/jeb.00848

Carr ZR, Chen C, Ringuette MJ (2013) Finite-span rotating wings: three-dimensional vortex formation and variations with aspect ratio. Exp Fluids 54(2):1444. https://doi.org/10.1007/s0034 8-012-1444-8

Combes SA, Daniel TL (2003) Flexural stiffness in insect wings I. Scaling and the influence of wing venation. J Exp Biol 206(17):2979_ 2987. https://doi.org/10.1242/jeb.00523

Ellington CP, van den Berg C, Willmott AP, Thomas ALR (1996) Leading-edge vortices in insect flight. Nature 384(6610):626-630. https://doi.org/10.1038/384626a0

Garmann DJ, Visbal MR (2014) Dynamics of revolving wings for various aspect ratios. J Fluid Mech 748:932-956. https://doi. org/10.1017/jfm.2014.212

Garmann DJ, Visbal MR, Orkwis PD (2013) Three-dimensional flow structure and aerodynamic loading on a revolving wing. Phys Fluid 25(3):034101. https://doi.org/10.1063/1.4794753

Graftieaux L, Michard M, Grosjean N (2001) Combining PIV, POD and vortex identification algorithms for the study of unsteady turbulent swirling flows. Meas Sci Technol 12(9):1422-1429. https ://doi.org/10.1088/0957-0233/12/9/307

Harbig RR, Sheridan J, Thompson MC (2013) Reynolds number and aspect ratio effects on the leading-edge vortex for rotating insect wing planforms. J Fluid Mech 717:166-192. https://doi. org/10.1017/jfm.2012.565

Hunt JCR, Wray AA, Moin P (1988) Eddies, stream, and convergence zones in turbulent flows. Center for Turbulence Research Report CTR-S88, pp 193-208

Jardin T (2017) Coriolis effect and the attachment of the leading edge vortex. J Fluid Mech 820:312340. https://doi.org/10.1017/ jfm.2017.222

Jardin T, David L (2014) Spanwise gradients in flow speed help stabilize leading-edge vortices on revolving wings. Phys Rev E 90(1):013011. https://doi.org/10.1103/PhysRevE.90.013011

Jardin T, David L (2015) Coriolis effects enhance lift on revolving wings. Phys Rev E 91(3):031001. https://doi.org/10.1103/PhysR evE.91.031001

Jones AR, Babinsky H (2011) Reynolds number effects on leading edge vortex development on a waving wing. Exp Fluids 51(1):197-210. https://doi.org/10.1007/s00348-010-1037-3

Jones AR, Manar F, Phillips N, Nakata T, Bomphrey R, Ringuette MJ, Percin M, van Oudheusden B, Palmer J (2016a) Leading edge vortex evolution and lift production on rotating wings. In: 54th AIAA aerospace sciences meeting, AIAA SciTech Forum, San Diego. https://doi.org/10.2514/6.2016-0288

Jones AR, Medina A, Spooner H, Mulleners K (2016b) Characterizing a burst leading-edge vortex on a rotating flat plate wing. Exp Fluid 57(4):1-16. https://doi.org/10.1007/s00348-016-2143-7

Lentink D, Dickinson MH (2009) Rotational accelerations stabilize leading edge vortices on revolving fly wings. J Exp Biol 212(16):2705-2719. https://doi.org/10.1242/jeb.022269

Moffatt HK (1969) The degree of knottedness of tangled vortex lines. J Fluid Mech 35(1):117-129. https://doi.org/10.1017/S002211206 9000991

van Oudheusden BW (2013) PIV-based pressure measurement. Meas Sci Technol 24(3):032001. https://doi.org/10.1088/09570233/24/3/032001

Ozen CA, Rockwell D (2012) Flow structure on a rotating plate. Exp Fluid 52(1):207-223. https://doi.org/10.1007/s00348-011-1215-y

Percin M (2015) Aerodynamic mechanisms of flapping flight. PhD thesis, Delft University of Technology. https://doi.org/10.4233/ uuid:4d535d87-d11e-4916-9143-5e6762c56152 
Percin M, van Oudheusden BW (2015a) Flow visualization and force measurements on accelerated revolving flat plates at low Reynolds numbers. In: 45th AIAA fluid dynamics conference, Dallas, Texas, USA, pp 1-12. https://doi.org/10.2514/6.2015-3074

Percin M, van Oudheusden BW (2015b) Three-dimensional flow structures and unsteady forces on pitching and surging revolving flat plates. Exp Fluid 56(2):47. https://doi.org/10.1007/s0034 8-015-1915-9

Percin M, Hu Y, van Oudheusden BW, Remes B, Scarano F (2011) Wing Flexibility effects in Clap-and-Fling. Int J Micro Air Veh 3(4):217-228. https://doi.org/10.1260/1756-8293.3.4.217

Pines DJ, Bohorquez F (2006) Challenges facing future micro-airvehicle development. J Aircraft 43(2):290-305. https://doi. org/10.2514/1.4922

Pitt Ford CW, Babinsky H (2013) Lift and the leading-edge vortex. J Fluid Mech 720:280-313. https://doi.org/10.1017/jfm.2013.28

Poelma C, Dickson WB, Dickinson MH (2006) Time-resolved reconstruction of the full velocity field around a dynamically-scaled flapping wing. Exp Fluid 41(2):213-225. https://doi.org/10.1007/ s00348-006-0172-3

Sane SP (2003) The aerodynamics of insect flight. J Exp Biol 206(23):4191-4208. https://doi.org/10.1242/jeb.00663

Shyy W, Aono H, Chimakurthi SK, Trizila P, Kang CK, Cesnik CES, Liu H (2010) Recent progress in flapping wing aerodynamics and aeroelasticity. Prog Aeronaut Sci 46(7):284-327. https://doi. org/10.1016/j.paerosci.2010.01.001
Tronchin T, David L, Farcy A (2015) Loads and pressure evaluation of the flow around a flapping wing from instantaneous $3 \mathrm{D}$ velocity measurements. Exp Fluid 56(1):7. https://doi.org/10.1007/s0034 8-014-1870-x

Usherwood JR, Ellington CP (2002) The aerodynamics of revolving wings I. Model hawkmoth wings. J Exp Biol 205(11):1547-1564

Vanyo JP (1993) Rotating fluids in engineering and science, 1st edn. Butterworth-Heinemann, Oxford

Venkata SK, Jones AR (2013) Leading-edge vortex structure over multiple revolutions of a rotating wing. J Aircraft 50(4):1312-1316. https://doi.org/10.2514/1.C032128

Zhao L, Huang Q, Deng X, Sane SP (2009) Aerodynamic effects of flexibility in flapping wings. J R Soc Interface. https://doi. org/10.1098/rsif.2009.0200

Zhao L, Deng X, Sane SP (2011) Modulation of leading edge vorticity and aerodynamic forces in flexible flapping wings. Bioinspir Biomim 6(3):036007. https://doi.org/10.1088/1748-3182/6/3/036007

Publisher's Note Springer Nature remains neutral with regard to jurisdictional claims in published maps and institutional affiliations. 